CERN-EP/87-140

6 August 1987

\title{
A PRECISE DETERMINATION OF THE ELECTROWEAK MIXING ANGLE FROM SEMILEPTONIC NEUTRINO SCATTERING
}

\author{
The CHARM Collaboration
}

J.V. Allaby, U. Amaldi, G. Barbiellini, M. Baubillier ${ }^{1)}$, F. Bergsma, A. Capone, W. Flegel, F. Grancagnolo ${ }^{2)}$, L. Lanceri ${ }^{3)}$, M. Metcalf, C. Nieuwenhuis, R. Pain ${ }^{4)}$, J. Panman, R. Plunckett ${ }^{5)}$ and K. Winter

CERN, Geneva, Switzerland

I. Abt, J. Aspiazu, A. Bungener, F.W. Büsser, P.D. Gall, T. Hebbeker, F. Niebergall, P. Schütt and P. Stähelin

II. Institut für Experimentalphysik ${ }^{6}$, Universität Hamburg, Hamburg, Fed. Rep. Germany

P. Gorbunov, E. Grigoriev, V. Khovansky and A. Rosanov

Institute for Theoretical and Experimental Physics, Moscow, USSR

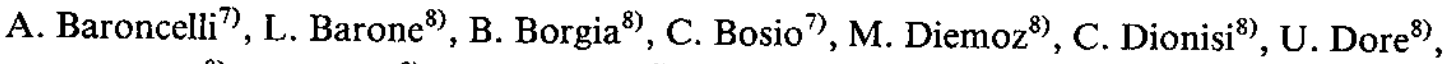
F. Ferroni ${ }^{8)}$, E. Longo ${ }^{8)}$, P.F. Loverre ${ }^{8)}$, L. Luminari ${ }^{8)}$, P. Monacelli ${ }^{8)}$, S. Morganti ${ }^{8)}$, F. de Notaristefani ${ }^{8)}$, C. Santoni ${ }^{9)}$, L. Tortora ${ }^{7)}$ and V. Valente ${ }^{10)}$

Istituto Nazionale di Fisica Nucleare, Rome, Italy

(Submitted to Zeitschrift für Physik)

1) Now at LPNHE, Paris, France.

2) Now at Dipartimento di Fisica, Università di Napoli and INFN Sezione di Napoli, Napoli, Italy.

3) Now at Dipartimento di Fisica, Università di Trieste and INFN Sezione di Trieste, Trieste, Italy.

4) On leave of absence from LPNHE, Paris, France.

5) Now at Physics Department, Rockefeller University, New York, USA.

6) Supported by Bundesministerium für Forschung und Technologie, Bonn, Fed. Rep. Germany, under contract 4HH22P.

7) INFN Sezione Sanità and Istituto Superiore di Sanità, Rome, Italy.

8) Dipartimento di Fisica, Università 'La Sapienza', Roma and INFN Sezione di Roma, Rome, Italy.

9) Now at Institute of Physics, University of Basel, Switzerland.

10) Laboratori Nazionali INFN Frascati, Italy. 


\begin{abstract}
The cross-section ratio of neutral-current and charged-current semileptonic interactions of muon-neutrinos on isoscalar nuclei has been measured with the result: $R^{\nu}=0.3093 \pm 0.0031$ for hadronic energy larger than $4 \mathrm{GeV}$. From this ratio we determined the electroweak mixing angle $\sin ^{2} \theta_{\mathrm{w}}=0.236+0.012\left(\mathrm{~m}_{\mathrm{c}}-1.5\right) \pm 0.005$ (exp.) \pm 0.003 (theor.), where $\mathrm{m}_{\mathrm{c}}$ is the charm-quark mass in $\mathrm{GeV} / \mathrm{c}^{2}$. Comparison with direct measurements of $\mathrm{m}_{\mathrm{W}}$ and $\mathrm{m}_{Z}$ determines the radiative shift of the intermediate boson mass $\Delta \mathrm{r}=0.077 \pm 0.025$ (exp.) \pm 0.038 (syst.), in agreement with the prediction. Assuming the validity of the electroweak standard theory we determined $\varrho=0.990-0.013$ $\left(\mathrm{m}_{\mathrm{c}}-1.5\right) \pm 0.009$ (exp.) \pm 0.003 (theor.).
\end{abstract}




\section{INTRODUCTION}

The Standard Model of the electroweak gauge theory is successful in describing a large amount of experimental data [1]. Parity violation in atomic transitions [2], the asymmetry in the scattering of polarized electrons on deuterium [3], the neutral-current- induced elastic scattering of muonneutrinos on electrons [4, 5] and on protons [6], deep inelastic semileptonic neutrino scattering by the neutral weak current $[7,8]$, and the direct observation of the weak bosons $[9,10]$ are all consistent with universal strength of the forces associated with the SU(2) and $U(1)$ symmetry groups of the standard model.

The fundamental quantities of the Standard Model, $g$ and $g^{\prime}$, and the masses of the weak bosons, $\mathrm{m}_{\mathrm{W}}$ and $\mathrm{m}_{\mathrm{Z}}$, are related to the angle $\theta_{\mathrm{w}}$ that describes the mixing of the two local symmetries through the relations

$$
\begin{aligned}
\tan \theta_{\mathrm{w}} & =\mathrm{g}^{\prime} / \mathrm{g} \\
\sin ^{2} \theta_{\mathrm{w}} & =1-\mathrm{m}_{\mathrm{w}}^{2} / \mathrm{m}_{\mathrm{Z}}^{2} .
\end{aligned}
$$

The value of this mixing parameter is not predicted by the model. It can be determined experimentally from measurements of the processes mediated by the neutral weak current. The values thus determined from various types of experiment are shifted by electroweak radiative corrections which have to be taken into account to perform a precise test of the theory. Because of the gauge nature of the interaction these radiative corrections can be calculated to all orders. Table 1 summarizes measured and corrected values of $\sin ^{2} \theta_{\mathrm{w}}$ up to 1985. The agreement of the values corrected for electroweak radiative effects is excellent, whereas the measured values differ from each other.

A more advanced test of the theory, at the level of the radiative corrections of order $\alpha$, can be performed by a precise determination of $\sin ^{2} \theta_{w}$ from semileptonic neutrino scattering and the direct measurements of $\mathrm{m}_{\mathrm{w}}$ and $\mathrm{m}_{\mathrm{Z}}$. The Fermi interaction, mediated by the weak bosons, leads to a relation between the fine-structure constant $\alpha$, the Fermi coupling constant $G_{F}$, the boson masses, and the weak mixing parameter $\sin ^{2} \theta_{\mathrm{w}}$

$$
\mathrm{m}_{\mathrm{W}}^{2}=\mathrm{m}_{\mathrm{Z}}^{2} \cos ^{2} \theta_{\mathrm{w}}=\pi \alpha / \sqrt{ } 2 \mathrm{G}_{\mathrm{F}} \sin ^{2} \theta_{\mathrm{w}} .
$$

Using $1 / \alpha=137.035963(15)$, as measured by the Josephson effect, and $G_{F}^{\mu}=1.16637(2) \times$ $10^{-5} \mathrm{GeV}^{-2}$ as determined from the muon lifetime, leads in lowest order to the relations:

$$
\begin{aligned}
\sin ^{2} \theta_{\mathrm{w}}^{\text {unc }} & =\left(37.281 \mathrm{GeV} / \mathrm{m}_{\mathrm{w}}\right)^{2} \\
\sin ^{2} 2 \theta_{\mathrm{w}}^{\text {unc }} & =\left(74.562 \mathrm{GeV} / \mathrm{m}_{\mathrm{Z}}\right)^{2} .
\end{aligned}
$$

Because of the definition of $\sin ^{2} \theta_{\mathrm{w}}$ in eq. (2) this relation must be radiatively corrected by using values of $\alpha$ and $\mathrm{G}_{\mathrm{F}}$ at the mass of $\mathrm{m}_{\mathrm{w}}$. The change of the value of $\mathrm{G}_{\mathrm{F}}\left(\mathrm{m}_{\mathrm{w}}^{2}\right)$ is negligible, whereas $\alpha$ is changed to $\alpha\left(\mathrm{m}_{\mathrm{w}}\right)=\alpha(0) /(1-\Delta \mathrm{r})$ with

$$
\Delta \mathrm{r}=0.0713 \pm 0.0013
$$

assuming $\mathrm{m}_{\text {Higgs }}=100 \mathrm{GeV}$ and a top quark mass of $\mathrm{m}_{\mathrm{t}}=45 \mathrm{GeV}[1,11]$. The predicted corresponding radiative shift of the $\mathrm{W}$ mass is about $3 \mathrm{GeV}$. The radiative correction can be experimentally determined from the measurements of $m_{w}$ and $m_{z}$ and of $\sin ^{2} \theta_{w}$ through the relation

$$
\Delta \mathrm{r}=1-\left(\pi \alpha / \mathrm{m}_{\mathrm{W}}^{2} \sqrt{2} \mathrm{G}_{\mathrm{F}} \sin ^{2} \theta_{\mathrm{W}}\right)
$$

and the corresponding relation involving $\mathrm{m}_{\mathrm{Z}}$. A comparison with the calculated radiative shift thus tests the predictive power of the Standard Model at the level of first-order radiative corrections in the same way as the predictive power of QED has been probed on the Lamb shift and on $(g-2)$ of the 
muon and the electron. The electroweak radiative shift is approximately 50 times larger than $(\mathrm{g}-2)$ of the muon, owing to the larger masses of the weak bosons involved.

We report here on a precise determination of $\sin ^{2} \theta_{\mathrm{w}}$ from semileptonic neutrino scattering ${ }^{*}$ which allows this test to be performed at a sensitive level. The result puts constraints on new physics which might be appended to the Standard Model, on the mass of the top quark and of a hypothetical fourth generation charged lepton as well as on the mass of additional neutral gauge bosons. Furthermore, this measurement now provides an accurate value of $\sin ^{2} \theta_{\mathrm{w}}$ which can be used to rule out, or at least constrain, Grand Unified Theories which predict it.

\section{DETERMINATION OF $\sin ^{2} \theta_{\mathrm{w}}$ FROM SEMILEPTONIC DEEP INELASTIC $\nu_{\mu}$ SCATTERING}

Several methods can be used to determine a value of $\sin ^{2} \theta_{\mathrm{w}}$ from semileptonic neutrino scattering. We have used a method recently proposed by Llewellyn Smith [12]. The coupling of the neutral weak current to fermions of charge $q$ and the third component of weak isospin $\mathrm{I}_{3}$ is according to the Standard Model:

$$
\begin{gathered}
\mathrm{g}_{\mathrm{L}}=2\left(\mathrm{I}_{3}-\mathrm{q} \sin ^{2} \theta_{\mathrm{w}}\right) \\
\mathrm{g}_{\mathrm{R}}=-2 \mathrm{q} \sin ^{2} \theta_{\mathrm{w}} .
\end{gathered}
$$

For the constituent $\mathrm{u}$ and $\mathrm{d}$ valence quarks of nucleons we thus expect

$$
\begin{aligned}
& \mathrm{g}_{\mathrm{L}}^{\mathrm{u}}=1-4 / 3 \sin ^{2} \theta_{\mathrm{w}}, \quad \mathrm{g}_{\mathrm{L}}^{\mathrm{d}}=-1+2 / 3 \sin ^{2} \theta_{\mathrm{w}}, \\
& \mathrm{g}_{\mathrm{R}}^{\mathrm{u}}=-4 / 3 \sin ^{2} \theta_{\mathrm{w}}, \quad \mathrm{g}_{\mathrm{R}}^{\mathrm{d}}=\quad 2 / 3 \sin ^{2} \theta_{\mathrm{w}} .
\end{aligned}
$$

Llewellyn Smith has shown that for isoscalar target nuclei the contribution of $\mathrm{u}, \overline{\mathrm{u}}$, and $\mathrm{d}, \overline{\mathrm{d}}$ quarks to the cross-sections of deep inelastic scattering of neutrinos by the neutral weak current (NC) and the charged weak current (CC) are related to $\sin ^{2} \theta_{\mathrm{w}}$ by the relation

$$
\begin{aligned}
\mathrm{d}^{2} \sigma_{\mathrm{NC}}^{\nu(\tilde{v})} / \mathrm{dxdy} & =\left(1 / 2-\sin ^{2} \theta_{\mathrm{w}}+5 / 9 \sin ^{4} \theta_{\mathrm{w}}\right) \mathrm{d}^{2} \sigma_{\mathrm{C}}^{\nu(\tilde{\nu})} / \mathrm{dxdy} \\
& +5 / 9 \sin ^{4} \theta_{\mathrm{w}} \mathrm{d}^{2} \sigma_{\mathrm{CC}}^{\vec{\nu}(\nu)} / \mathrm{dxdy} .
\end{aligned}
$$

Its validity depends on weak isospin symmetry. Integrating Eq. (9) over $x$ and $y$, one obtains a relation containing only ratios of cross-sections,

$$
\mathrm{R}^{\nu}=\sigma_{\mathrm{NC}}^{\nu} / \sigma_{\mathrm{CC}}^{\nu}=1 / 2-\sin ^{2} \theta_{\mathrm{W}}+5 / 9 \sin ^{4} \theta_{\mathrm{W}}(1+\mathrm{r}),
$$

where $\mathrm{r}=\sigma_{\mathrm{CC}}^{\bar{\nu}} / \sigma_{\mathrm{CC}}^{\nu}$.

A precise measurement of $\mathrm{R}^{\nu}$ can be performed without the need to monitor precisely the incident neutrino flux. Events induced by neutrinos have to be classified as NC or CC events by the detection of a muon. One must, therefore, aim at a high efficiency of muon detection in designing a precise experiment. The track length of muons increases linearly with the mean neutrino energy and, hence, with parent (pion and kaon) beam momentum, whereas the length of hadron showers which may obscure the muon track increases only logarithmically with the beam momentum. Thus, a highmomentum parent beam is required. For a $160 \mathrm{GeV}$ beam momentum, which was chosen as an optimum for the event statistics and the muon detection efficiency, more than $96 \%$ of the muons originating in $\mathrm{CC}$ reactions can be recognized; the losses can be calculated precisely, allowing a total experimental error of $\Delta R / R= \pm 0.01$ to be reached. A measurement of the ratio $r$ of $C C$ cross-sections for antineutrino and neutrino deep inelastic scattering is required to determine the

*) See also J.V. Allaby et al. (CHARM Collaboration), Phys. Letters 177B, 446 (1986). 
correction term, $5 / 9 \times \sin ^{4} \theta \mathrm{w}$, which accounts for $\mathrm{NC}$ scattering on right-handed quarks. The experimental precision required for $\mathrm{r}$ is reduced by the coefficient $5 / 9 \sin ^{4} \theta_{\mathrm{W}} \approx 0.035$; hence a measurement error of $\Delta r / r= \pm 0.04$, because of the relative antineutrino and neutrino flux monitoring required, is sufficient to achieve a total experimental error of $\Delta \sin ^{2} \theta_{\mathrm{W}}= \pm 0.005$ at which we were aiming.

Equation (10) holds for deep inelastic scattering of neutrinos; processes which are not sensitive to the weak charge of the quarks, namely quasi-elastic scattering and coherent pion production, have to be rejected. This would be best achieved according to studies of deep inelastic scattering [13] by selecting events with a momentum transfer $\mathrm{Q}^{2}>4 \mathrm{GeV}^{2}$. In NC interactions a direct measurement of $\mathrm{Q}^{2}$ is not possible, because the outgoing neutrinos are undetectable. However, for these near elastic processes there is a close relation between $Q^{2}$ and the hadron energy $E_{h}$. A selection of events with $E_{h}>4 \mathrm{GeV}$ is an equivalent way of rejecting events which are not due to deep inelastic neutrino scattering.

Some processes contributing to deep inelastic scattering do break the isospin symmetry required for the validity of Eq. (10). These are mainly due to the asymmetry of the strange-quark sea and the charm-quark sea, owing to their different mass values, and to quark mixing. These asymmetries, amounting to $\Delta \mathbf{R}^{v} / \mathbf{R}^{\nu} \approx 2 \%$, have to be corrected for by using the quark model to determine $\sin ^{2} \theta_{\mathrm{w}}$. The remaining uncertainties due to our present limited knowledge of the quark structure of nucleons have been estimated to contribute $\Delta \sin ^{2} \theta_{\mathrm{w}}= \pm 0.005$, the largest uncertainty being in the choice of the charm-quark mass which is required to describe the threshold of charm production in CC interactions. However, these uncertainties are expected to be reduced once better data are available to describe the quark structure of the nucleon.

The experimental set-up, consisting of the neutrino beam and the neutrino detector, and the data taking, trigger, and calibration procedure are described in Section 3. In Section 4 the analysis of the data, the determination of background, the classification of events, and the corrections applied to these classes are described. The results obtained after quark-model and radiative corrections have been applied, and their comparison with the results on $\mathrm{m}_{\mathrm{W}}$ and $\mathrm{m}_{\mathrm{Z}}$ obtained at the CERN $\mathrm{p} \overline{\mathrm{p}}$ collider, are described in Section 5. Their impact on physics is discussed in Section 6.

\section{EXPERIMENTAL SET-UP}

\subsection{The narrow-band neutrino beam}

The data were obtained in 1984 during a high-statistics exposure of the CHARM detector in the narrow-band neutrino beam at the CERN $450 \mathrm{GeV}$ Super Proton Synchrotron (SPS). Neutrinos are produced in the decay of charge- and momentum-selected pions and kaons. Particles of positive or of negative charge produce a pure neutrino or antineutrino beam with a well-known energy spectrum. A small background, predominantly of low energy, the so-called wide-band (WB) background, is due to decays of particles which do not pass the charge and momentum selection of the beam line.

A $160 \mathrm{GeV}$ narrow band beam (NBB), developed by Grant and Maugain [14] as a high-flux version of the CERN NBB optics, was used. The event rate per incident proton has been increased by about $50 \%$ by increasing the effective momentum bite of the beam with respect to the previous version of the NBB. The beam characteristics satisfy the conditions for a precision measurement of $\mathrm{R}^{\nu}$, namely [15]:

i) A high average neutrino energy, needed for efficient muon recognition in the CHARM detector;

ii) low background contributions, measurable with high accuracy;

iii) the possibility of measuring the relative $\bar{\nu} / \nu$ flux normalization accurately;

iv) calculable neutrino energy spectrum;

v) high event rate. 
The corresponding neutrino energy spectrum in the fiducial volume of the detector for accepted events is shown in Fig. 1. The contributions of pion and kaon decays are distinctly separated.

The elements of the beam line are shown schematically in Fig. 2 . The beam travels in vacuum and is nearly parallel on entering the $300 \mathrm{~m}$ long decay tunnel. The vacuum is only interrupted at the place of a Cherenkov counter.

The components of the beam can be separated into four regions:

i) The target region, where secondary hadrons are produced by a $450 \mathrm{GeV}$ proton beam incident on a $3 \mathrm{~cm}$ diameter carbon target.

ii) The secondary hadron beam line of about $120 \mathrm{~m}$ length, where hadrons are selected by sign and momentum and focused towards the decay region. The beam line was tuned to a central momentum of $160 \mathrm{GeV} / \mathrm{c}$, with a momentum bite of $\pm 9 \%$ (r.m.s.); the central momentum is known to about $3 \%$. At the end of the beam line the angular divergence is $\pm 0.40 \mathrm{mrad}$ in the horizontal plane and $\pm 0.48 \mathrm{mrad}$ in the vertical plane.

iii) About $8 \mathrm{~m}$ behind the last quadrupole magnet, hadrons enter a $292 \mathrm{~m}$ long decay tunnel, which was evacuated to a pressure of 0.15 Torr. The entrance window of the decay region is made of a $0.2 \mathrm{~mm}$ thick titanium membrane to minimize interactions of hadrons.

iv) At the end of the decay tunnel all surviving hadrons are absorbed in the first few metres of a $185 \mathrm{~m}$ long iron shield. Muons are ranged out by a combination of the iron shield and a $220 \mathrm{~m}$ long earth shielding following it.

To avoid any losses of particles due to wrong steering of the beam, the onset of such bad conditions was detected by monitors surrounding the vacuum pipe. Various intensity monitors for both primary and secondary particles were placed along the beam line. The intensity of the ejected proton beam was measured by a beam-current transformer and the beam position on the $3 \mathrm{~cm}$ diameter carbon target was carefully monitored using a secondary emission chamber mounted ahead of the target. Another secondary emission chamber, downstream from the target, monitored the secondaries produced. Two identical beam-current transformers placed just in front of the entrance of the decay tunnel, after the last quadrupole lens, were used to measure the intensity of the secondary beam. The relative amounts of pions and kaons in the beam were determined experimentally using a helium-filled differential Cherenkov counter.

The beam could be closed by an iron dump just in front of the decay tunnel. Data were taken in this condition to determine experimentally the neutrino and antineutrino fluxes from decays ahead of the dump, the so-called WB background. To avoid background created by proton interactions in vacuum windows downstream from the dump which cannot be calculated well, the dump was placed inside an area which was part of the decay tunnel vacuum. The procedure used to correct for the WB background is described in Section 4.

The flux of muons originating from pion and kaon decays was measured by a system of solid-state detectors inserted into narrow gaps within the iron shield. They were also used to monitor the beam position and profile.

Pulse trains from scintillation counters located in the gaps of the iron shield were folded with the busy signals of the neutrino detector to determine the loss of neutrino events due to dead-time.

\subsection{The CHARM detector}

The CHARM neutrino detector is composed of a fine-grain calorimeter surrounded by a magnetized iron frame and of a muon spectrometer. The calorimeter has a sampling step corresponding to one radiation length or 0.22 pion absorption lengths, with scintillators, proportional drift tubes, and streamer tubes, as detecting elements. It is described in detail elsewhere [16]. 
The response of the calorimeter was calibrated in electron and pion beams in the range from 0.5 to $140 \mathrm{GeV} / \mathrm{c}$ [17]. The energy of hadron showers is measured by the scintillators with a resolution of

$$
\sigma(\mathrm{E}) / \mathrm{E}=0.487 / \sqrt{(\mathrm{E} / \mathrm{GeV})}+0.0127
$$

The lateral vertex position of hadron showers is determined by combining the measurements of all detection elements. The resolution is given in Fig. 3; it reaches a precision of better than $3 \mathrm{~cm}$ above $50 \mathrm{GeV}$ [17]. The muon momentum is measured by magnetic deflection; the resolution is $\sigma(\mathrm{p}) / \mathrm{p} \approx$ $15 \%$ at low energies and increases to $20 \%$ at $150 \mathrm{GeV} / \mathrm{c}$.

For this experiment the fiducial volume extended over 55 target plates, leaving five plates at the beginning of the detector to reject incoming tracks and 18 plates at the end to ensure good shower containment. Laterally it extended over a surface of $240 \times 240 \mathrm{~cm}^{2}$. This resulted in a fiducial mass of $87 \mathrm{t}$.

\subsubsection{Data taking}

The detector was exposed to neutrino and antineutrino beams; part of the time a dump block was inserted into the beam to determine the WB background.

The number of protons on target (p.o.t.) collected for each of these settings is given in Table 2. Additional data were taken with the Cherenkov counter in the beam to measure the pion and kaon composition.

Protons were extracted from the SPS in bursts with a bell-shaped time structure, approximately $500 \mu \mathrm{s}$ wide at the base. This spill-width was chosen as a compromise between excessive detector dead-time on the one hand and a large cosmic-ray background on the other hand. A $750 \mu$ s gate (GATE 1) was used at the ejection-time to include the spill-width and small drifts in the timing of the ejection.

In addition to this gate covering the extraction-time, events were also accepted in a second gate (GATE 2), which was opened after the end of the proton spill. GATE 2 was 3 times longer than GATE 1 and provided a measurement of the cosmic-ray background in the neutrino triggers. This gating structure was repeated for every SPS cycle with a cycle time of $12 \mathrm{~s}$.

\subsubsection{Trigger}

The trigger logic was based on scintillator information with a discriminator threshold set to $1 / 5$ of the average energy deposition of a minimum ionizing particle.

The trigger requires at least one hit recorded in at least four scintillator planes with the requirement of no hit in the first plane to veto incoming charged particles. This condition corresponds to a minimum energy deposition of $1.5 \mathrm{GeV}$ for showers. The efficiency of the trigger will be discussed in a later section.

\subsubsection{Selection of valid bursts}

Beam-monitor information was made available to the on-line system in the form of direct pulse trains ('hardware information') and was also transmitted through the Neutrino Facility Monitor computers in the form of blocks of data ('software information').

Software information was used to define conditions for rejecting bad quality bursts. The thresholds for these cuts were fixed for long periods during which the running conditions were stable.

Intensity and position of the extracted proton beam as well as of the secondary hadron beam were thus constrained to stay within acceptable limits. Bursts with insufficient monitoring information (mainly due to failures in data transfer) were also rejected. 
The percentage of protons on target rejected after the cuts for each running condition and the percentage effectively used in the analysis are given in Table 2 .

\subsubsection{Suppression of 'double events'}

The process of digitization of events in the proportional drift-tube system has an active gate length of $\approx 1 \mu \mathrm{s}$. Because of this gate width a few per cent of the events occur together with a second event within the same conversion-time.

For each event the time which elapsed between the trigger and the next interaction was recorded with a time-to-digital converter (TDC). The time measurement was started $200 \mathrm{~ns}$ after the trigger and was stopped by the following interaction which released a total energy larger than $\approx 1.5 \mathrm{GeV}$. The time was digitized in $50 \mathrm{~ns}$ intervals. Events followed by another interaction in a time window of 200 to $1200 \mathrm{~ns}$ were rejected in the analysis. Most of the problems due to pattern recognition introduced by overlay events were removed in this way.

The procedure introduces an additional 'dead-time' of $\approx 2 \%$, which, however, does not affect the measurement of $\mathbf{R}^{\nu}$. This dead-time was determined by the fraction of events with TDC counts in a time window from 1200 to $2200 \mathrm{~ns}$ (see Fig. 4). Since the spill structure is nearly constant on a microsecond scale, the number of events rejected in the window from 200 to 1200 ns should be equal to the number of events found in a window of equal width following it. The data presented in Fig. 4 shows that the recognition efficiency for $\mathrm{CC}$ events is significantly affected by the presence of a second interaction; the event rate is normally expected to be flat in $\Delta \mathrm{t}$, as in the case for $\Delta \mathrm{t}>1.2 \mu \mathrm{s}$. The inclusion of the events occurring in the region labelled 'rejected' would therefore introduce an error in the measurement of $\mathrm{R}^{v}$.

\subsubsection{Relative Normalization of Different Running Conditions}

In order to subtract cosmic-ray background the exposures during GATE 2 had to be normalized relative to the respective exposures during GATE 1 for the four data-taking conditions. This normalization is performed by measuring the integrated gate length with a clock, taking the detector dead-time into account.

The subtraction of WB background requires a relative normalization of normal running compared to 'dump' running. This normalization is done on the basis of the integrated intensity of the secondary hadron beam, measured just ahead of the entrance of the decay tunnel. The intensity is measured with the beam-current transformers (BCTs) described before. The dead-time of the neutrino detector was taken into account weighted with the spill-time structure of the proton extraction. A small correction was applied for protons extracted at times outside the neutrino detector gate, but measured in the BCT gate ('gate inefficiency').

The relative normalization of the neutrino exposure with respect to the antineutrino exposure was performed with the help of a measurement of the integrated muon flux in the shielding obtained with the system of solid-state detectors (SSDs). Dead-time corrections weighted with the spill-time structure and corrections for 'gate inefficiency' were applied. The relative muon fluxes are closely related to the relative neutrino fluxes; only small corrections have to be made. Had the measured secondary hadron beams been chosen for this purpose larger uncertainties would have been introduced by the need to correct for the proton, pion, and kaon fractions in the positive and negative secondary beams.

After subtraction of the background muons coming from $\pi$ and $\mathrm{K}$ decays upstream of the decay tunnel ('trapped muons'), the SSDs measure the sum of the signal of muons produced by $\pi$ and $K$ decays. The ratio of the SSD signals in $\nu$ and $\bar{\nu}$ running determines the relative pion and kaon rates in these exposures; the ratios $\mathrm{K}^{+} / \pi^{+}$and $\mathrm{K}^{-} / \pi^{-}$were determined by a Cherenkov counter and the acceptance of the counters for measuring a muon from pion and kaon decay in the beam was 
calculated by Monte Carlo simulation. The sum of the signals of a system of detectors placed on a ring with $15 \mathrm{~cm}$ radius around the nominal beam axis at a depth of $30 \mathrm{~m}$ of iron was used for this normalization. This procedure minimizes the effects of variations in the steering of the beam. From the relative pion and kaon fluxes in the two exposures the neutrino to antineutrino flux ratio can be obtained. The acceptance for measuring muons in the counters was calculated with a Monte Carlo program, simulating the NBB line to obtain the phase-space densities of pions and kaons and of the daughter particles produced in their decays. The muons were tracked through the iron shielding and the acceptance for their detection with the SSDs was determined. The neutrinos were followed up to the neutrino detector to determine the corresponding neutrino flux ratio. The uncertainties in the determination of the ratio of the antineutrino and the neutrino fluxes are listed in Table 3.

\subsubsection{Dead-time correction}

The dead-time of the detector was measured using a $1 \mathrm{MHz}$ clock during the active time of the detector and during the integrated gate-time. This procedure gives an accurate sampling of the unweighted dead-time needed for subtraction of cosmic-ray background.

The flux-weighted dead-time was measured using signals from small scintillators inserted into the muon shield after $\approx 120 \mathrm{~m}$ of iron. These scintilators had a cross-section of $0.1 \mathrm{~cm}^{2}$ perpendicular to the beam and counted the beam related muon flux at a rate of about $100 \mathrm{kHz}$. The signals of these scintillators were counted during the active time of the detector (A), during the neutrino detector gate $(\mathrm{G})$, and during a wide gate $(\mathrm{W})$, around the proton extraction time. The probability of recording a muon signal in these scintillator counters is proportional to the neutrino flux at that time; therefore the use of these signals makes it possible to measure the flux-weighted dead-times. The expression $1-\mathrm{A} / \mathrm{G}$ determines the flux-weighted detector dead-time, and $1-\mathrm{G} / \mathrm{W}$ the 'gate inefficiency'.

Average weighted and unweighted dead-times are reported in Table 2; the effective dead-time introduced by the procedure used to reject double events is also given.

\subsubsection{Fast cosmic-ray filter}

In order to reduce the background of cosmic-ray events and consequently to reduce the statistical error when subtracting the background, a filter program was used, which consisted of two stages.

The first stage removed $\approx 80 \%$ of the cosmic-ray events, which were those with tracks entering the detector from the front face or from the side faces travelling in the beam direction; it was checked that it did not reject neutrino-induced events. Tracks were defined by hits in the proportional tubes and allowance was made for one extra noise hit.

The second stage was more sophisticated and after this stage only one out of 2000 cosmic-ray triggers survived, reducing the trigger rate of $4 \mathrm{kHz}$ to a few per second. This rejection rate is achieved by an efficient suppression of tube hits which are not related to the event ('noise') and by the use of scintillator as well as proportional tube information. On the basis of local patterns, active elements are classified as 'shower-hits' or 'track hits'. Events with shower hits are not rejected. For the remaining events it was checked whether the hits, grouped in clusters, were connected to form an entering track. The cluster sizes were checked for consistency with the track angle. Such events were rejected too.

This algorithm has a small inefficiency for accepting neutrino-induced NC events, which will be quantified in the following section. 


\subsubsection{Trigger and filter inefficiencies}

A priori, a lower triggering efficiency has to be suspected for NC events compared to that for $\mathrm{CC}$ events, because of the absence of a charged primary lepton track. The potential loss is expected to increase for decreasing shower energy. To study this effect we used the observed $\mathrm{CC}$ event sample. A new sample of 'pseudo NC events' was created by removing the muon in each $\mathrm{CC}$ event. This procedure was performed by the code of the reconstruction program. Particular care was taken to recalculate the scintillator pulse heights by subtracting the expected muon contribution along the muon track. The calculated scintillator pulse heights were used to determine whether the 'pseudo NC' event satisfied the trigger requirements. The fraction of lost events per hadron energy bin is reported in Fig. 5.

The fraction of these pseudo NC events rejected by the cosmic-ray filter is also given in the figure. The correlation between the two effects is small and the total loss is approximately equal to the sum of the two individual losses. The combined inefficiency for NC events as a function of hadron energy is also shown in Fig. 5. For hadron energy above $4 \mathrm{GeV}$ the integrated inefficiency amounts to $(0.2 \pm 0.1) \times 10^{-3}$ for neutrino events and $(0.4 \pm 0.4) \times 10^{-3}$ for antineutrino events.

\subsubsection{Efficiency near the veto plane}

Back-scattering may have a different effect on the veto efficiency for $\mathrm{NC}$ and $\mathrm{CC}$ events. We determined that efficiency using $\mathrm{NC}$ and $\mathrm{CC}$ events produced in the centre of the fiducial volume. A sample of 'pseudo NC events' was created by shifting the longitudinal vertex coordinate (plane number) towards the veto plane. The reconstructed scintillator pulse heights were used to determine whether the 'pseudo NC event' satisfied the veto criteria. The ratio of events accepted by the veto condition is reported in Fig. 6 as a function of plane number for NC and CC events. Integrating the efficiency over the fiducial volume we find a difference for $\mathrm{CC}$ and $\mathrm{NC}$ events $<10^{-4}$. This difference has been taken into account in the analysis.

\section{ANALYSIS}

The analysis was performed in several steps. First, events were separated into classes of NC and $\mathrm{CC}$ candidates, then corrections were applied for event loss and background to obtain the physical $\mathrm{NC}$ and $\mathrm{CC}$ event numbers.

\subsection{Event recognition}

Interactions in the CHARM detector were classified by an automatic pattern-recognition program on an event-by-event basis. Selection criteria were applied which attempt to identify optimally the physical processes and to minimize the corrections needed to relate the visible cross-sections to the physical cross-sections.

\subsubsection{Classification criteria}

Neutrino interactions were defined as events which have no entering charged tracks.

A neutrino event is classified as a $\mathrm{CC}$ interaction if it contains a muon originating at the event vertex. A muon is assumed to originate from the event vertex if its extrapolated intercept with the vertex plane of the event lies within $30 \mathrm{~cm}$ of the measured shower vertex, or if sufficient energy is measured in a box around the extrapolated point. Muons have to be seen in the $140 \mathrm{~ns}$ scintillator gate following the trigger to ensure suppression of overlay tracks seen in the proportional-tube system, which has an effective gate length of $\approx 1 \mu \mathrm{s}$. The muon has to be visible over a range corresponding to an energy loss of at least $0.67 \mathrm{GeV}$. Its total range has to exceed $1 \mathrm{GeV}$ energy loss, corresponding to a penetration of 20 calorimeter planes. The visible range of a muon track is defined as the length, measured in equivalent range, between the first clearly visible point outside the shower 
and the last point on the track, as seen by the proportional-tube system. Tube hits are deemed to be clearly visible points on a track if they are separated laterally by at least 5 quiet tubes from their neighbouring hits ( $15 \mathrm{~cm}$ isolation criterion). In addition they are required to be isolated from other hits which do not belong to the muon track, in the longitudinal direction. These isolation criteria are much more strict than the ones used in a previous publication on the same subject [7] and tend to reject more tracks on the basis of the requirement of $0.67 \mathrm{GeV}$ visible range.

All other neutrino-event candidates are classified as NC interactions. Only those events with a vertex inside the fiducial volume and with shower energy of at least $4 \mathrm{GeV}$ were analysed further. It was checked that no bias was introduced by these cuts in the NC to CC event ratio. Representations of a typical NC and a typical CC event in the CHARM detector are shown in Fig. 7.

In order to obtain the $\mathrm{CC}$ and $\mathrm{NC}$ event numbers from the automatic classification, the following corrections have to be applied to the event numbers:

i) classification inefficiencies of the automatic reconstruction program,

ii) loss of muons not satisfying the selection criteria,

iii) $\pi$ and $\mathrm{K}$ decays in the shower faking a primary muon,

iv) $\nu_{\mathrm{e}}$ background produced by $\mathrm{K}_{\mathrm{e} 3}$ decays in the beam,

v) cosmic-ray background and WB background.

These corrections will be discussed in the following subsections.

\subsubsection{Classification inefficiency}

The program recognized and flagged event topologies for which the automatic procedure could fail. Events were selected for scanning before the energy and fiducial volume cuts and the cut on the 'double-event' TDC were applied in order to check the systematic biases introduced by them. Approximately $1 \%$ of all neutrino triggers after rejection of the cosmic-ray events by the filter were flagged as events for which it is difficult to reconstruct the topology automatically. Typical cases are:

i) tracks which fail the vertex extrapolation because of random association of shower hits to the track resulting in a bad straight-line extrapolation to the vertex;

ii) tracks for which the scintillator timing criteria are not fulfilled owing to geometrical or electronic inefficiencies.

iii) multiple track events for which the matching of the two projections of the primary muon is difficult;

iv) events with inconsistencies in the vertex-finding algorithm, producing two incompatible estimates for the vertex position.

These flagged events were visually inspected in a double scan and corrected on an event-by-event basis. Roughly 600 events out of a total of 4000 flagged events were reclassified. The scanning efficiency for this category of events was found to be $>99 \%$.

In addition to these flagged events a randomly selected sample totalling $5 \%$ of all triggers which survived the cosmic-ray filter were scanned in order to determine the inefficiency of the automatic pattern-recognition program and to correct for it on a statistical basis. The double scan showed that, after applying the energy and fiducial volume cuts, 7 events in this sample were misclassified (Table 4). A global correction for this classification error was then made; it introduced an uncertainty of $\Delta \mathrm{R}^{\nu} / \mathrm{R}^{\nu}<0.1 \%$.

\subsubsection{Muon-recognition losses}

Charged-current events in which the primary muon cannot be identified are by definition classified as NC. Some of these lost $\mathrm{CC}$ events have a muon with an energy of less than $1 \mathrm{GeV}$. Another contribution to the loss of $\mathrm{CC}$ events is caused by muons of more than $1 \mathrm{GeV}$ either leaving the sides of the detector before depositing sufficient energy, or being obscured by the hadronic 
shower. The two categories are of approximately equal importance. A correction is required for these $\mathrm{CC}$ losses. As this is the largest correction required, the precision which can be reached in measuring $\mathbf{R}^{\nu}$ depends in an essential way on the reliability of estimating these losses. To test our methods we determined the correction in two independent ways. Both methods do not require a simulation of hadronic showers, a notoriously difficult enterprise.

For the first method, real CC events were used in order to reproduce the properties of hadronic showers, and their primary muon tracks removed. Only events for which the primary track was well reconstructed were used in this analysis. This selection introduces a small bias in the shower-energy spectrum, which is corrected for in the subsequent analysis by calculating the correction as a function of the shower energy. In a second step, a simulated muon is merged with the original shower. The direction and momentum of the muon are chosen randomly in such a way as to agree statistically with the expected distribution from the NBB. The muon-reconstruction efficiency is obtained by passing these 'semi Monte Carlo' events through the standard analysis chain.

A second method was developed in which the hadronic shower properties were determined from $\mathrm{CC}$ events. The 'effective' length of showers was obtained by measuring for each recognized CC event the distance between the shower vertex and the first point of the muon track which is clearly visible outside the shower. Histograms were made of these distances as a function of the relevant kinematic variables, namely the shower energy and the opening angle between the muon and the shower. Typical shower-length distributions for 10 and $50 \mathrm{GeV}$ hadron energies are shown in Fig. 8.

These histograms were used to determine the invisible part of simulated muon tracks. These tracks were simulated taking all details of the detector into account and the selection criteria were then applied in order to determine the efficiency. The agreement between data and the pseudo Monte Carlo events is very good; Fig. $9 \mathrm{a}$ and $\mathrm{b}$ shows the comparison for the shower-energy distribution and for the distribution of event radius with respect to the beam axis. Comparisons of average hadron shower length with hadron shower energy (a) and measured muon total range distribution (b) are shown in Fig. 10.

The total inefficiency of the selection criteria for muons is calculated to be $3.5 \%$. Its variation with the generated muon momentum is shown in Fig. 11. The two methods give a consistent result to within $2 \%$ of the correction.

The uncertainty of the parent-beam-momentum and that of the muon-momentum measurement by the CHARM detector introduce systematic errors in the correction. In order to largely eliminate these systematic errors in the calculation of the correction we defined a 'reference region' of muon momentum and normalized lost muons to the event number found in this region. Charged-current events with a primary muon of 3 to $5 \mathrm{GeV}$ energy were accounted for in the reference region. The ratio of all $\mathrm{CC}$ events with an unseen muon and of identified $\mathrm{CC}$ events in the reference region was then calculated using the Monte Carlo program described above. The absolute number of $\mathrm{CC}$ events for which the muon was not recognized is obtained by multiplying this ratio with the actual number of $\mathrm{CC}$ events found in the reference region. Contributions from background events in the reference region were also taken into account. This procedure eliminates all errors introduced by uncertainties in the beam energy, the energy loss of muons, the density of the detector, and the slope of the y-distribution. This cancellation is exact for all such scale errors because the simulated events in the reference region scale with the number of events with lost muons. The remaining systematic error is dominated by the uncertainty in the determination of the shower length ( \pm 0.5 calorimeter plane) and is estimated to be $\pm 2.8 \%$ of the correction corresponding to $\Delta \mathrm{R}^{\nu} / \mathrm{R}^{\nu}=0.32 \%$. In the calculation of the effect of lost primary muons it was taken into account that in such $\mathrm{CC}$ events a secondary muon can be recognized as a primary muon (' $\pi / \mathrm{K}$-decay correction'). 


\subsection{4 $\pi$ and $K$ decays in showers and punch-through tracks}

A fraction of NC events containing a track which fulfils the requirements of a primary muon of a CC event are classified as CC events. This background is caused by decays of pions or kaons in the shower or by punch-through tracks.

We calculated this correction by using again events detected in the experiment. Charged-current events with well-measured primary muons were selected and the muon removed; the remainder of the event was then processed through the standard analysis chain. A second track fulfilling the conditions for a primary muon was searched for in these events.

The fraction of events for which a second muon was found then determined the correction to be applied. In the selected sample $[6.02 \pm 0.08$ (stat.) \pm 0.04 (syst.) $] \%$ of the events had a second track satisfying the primary-muon criteria. The systematic error includes biases introduced by the fact that in a small number of events the original primary muon cannot be perfectly removed.

However, the sample of showers obtained from CC events has some differences with respect to the NC events, which have to be taken into account. In a fraction of $\mathrm{CC}$ interactions, a second prompt muon in the shower comes from charm-quark production and subsequent semileptonic decay. Neutral-current interactions do not change the flavour of quarks and this effect is therefore absent in these interactions. The corresponding events must be subtracted from the event sample. This amounts to $(0.54 \pm 0.08) \%$ of the selected sample. Charm production from $\mathrm{d}$ and $\mathrm{s}$ quarks and its semileptonic decay into muons is modelled in a Monte Carlo program; the rate is determined by the ratio of dimuon and single-muon events produced by neutrinos found in the literature [18]. The model for charm production described thresholds by the so-called slow rescaling approximation with a charm-quark mass $\mathrm{m}_{\mathrm{c}}=1.5 \mathrm{GeV}$ [19]. The acceptance was calculated using a flat fragmentation function. The agreement with available data is shown in Fig. 12. An uncertainty of $\pm 15 \%$ is assumed for the absolute normalization of the subtraction (shaded areas in Fig. 12).

Strange particles are produced more frequently in NC events than in $\mathrm{CC}$ events. This leads to a larger contribution of decays of primary shower particles in NC events. The calculated correction is $(0.04 \pm 0.02) \%$.

In order to avoid biases introduced by the selection of the $\mathrm{CC}$ events used to determine the fraction of showers with two primary muons, the correction is applied as a function of the shower energy (Fig. 13). We also verified whether the selection of events, with well-measured muon tracks used to determine the correction did not introduce a change in the final fraction of events found with a second primary muon. However, a small contamination of the sample by NC events with a well-measured muon track has no effect on the final result.

The magnitude is significantly higher than that of the equivalent correction applied during the analysis of previous data [7], because in the present case no attempt was made to remove pion tracks from the primary muon sample on the basis of hadron-like behaviour.

\subsubsection{Electron-neutrinos produced in the $N B B$}

Both $\mathrm{NC}$ and $\mathrm{CC}$ interactions of electron-neutrinos originating from $\mathrm{K}_{\mathrm{e} 3}$-decays are classified as $\mathrm{NC}$ events. The contribution of these events amounts to $6.6 \%$ of the NC candidates in the $\nu$ exposure and $4 \%$ in the $\bar{\nu}$ exposure.

To calculate the correction, the ratio of kaons to pions in the beam had to be determined; it was measured using a focusing gas Cherenkov counter which was moved in the beam, near the hadron dump, for special runs. Figure 14 shows a typical Cherenkov pressure $(\mathrm{P})$ curve. The peaks in the region $0<\mathrm{P}<110$ matm, $135<\mathrm{P}<210$ matm and $450<\mathrm{P}<750$ matm correspond to $\pi$, $\mathrm{K}$, and p signals, respectively. The background was interpolated from the regions outside the peaks, assuming a linear variation. The measurements yielded the ratios: 


$$
\begin{aligned}
& \mathrm{K}^{+} / \pi^{+}=0.1282 \pm 0.0006 \text { (stat.) } \pm 0.0027 \text { (syst.) } \\
& \mathrm{p} / \pi^{+}=1.628 \pm 0.005 \text { (stat.) } \pm 0.070 \text { (syst.) } \\
& \mathrm{K}^{-} / \pi^{-}=0.0630 \pm 0.0011 \text { (stat.) } \pm 0.0030 \text { (syst.) } \\
& \overline{\mathrm{p}} / \pi^{-} \leq 1.3 \%(90 \% \mathrm{CL}) .
\end{aligned}
$$

The statistical errors are derived from the dispersion of the values obtained in different runs. The systematic errors are due to the uncertainties in the estimation of the background and in the definition of the $\pi$ and $K$ regions. The contamination of the pion peak by positrons and electrons was estimated to be $<0.87 \%$ and $<1.3 \%$ at $90 \% \mathrm{CL}$, respectively, for the $\nu$ and $\bar{\nu}$ beams and was subtracted.

The number of $\nu_{\mathrm{e}}$-induced events was calculated assuming e- $\mu$ universality of the weak interaction in the context of the Standard Model.

A small correction was applied for events with showers generating a decay muon, causing these events to be classified as CC events. Using the measurement of the $K / \pi$ ratio with an error of $\pm 3 \%$, this correction was calculated to $\pm 4 \%$ of its value, corresponding to $\Delta \mathrm{R}^{\nu} / \mathrm{R}^{\nu}=0.3 \%$.

\subsubsection{Wide-band background}

The neutrino flux from pion and kaon decays before the decay tunnel and before the momentum and charge selection is not easily calculable, and it is therefore preferable to measure it separately by blocking the entrance of the decay tunnel with a dump.

The procedure was to define all of the neutrino flux generated before the dump as background, and the flux from decays downstream of the dump as 'beam'. The relative normalization of the periods when the dump blocked the entrance of the decay tunnel with respect to the standard running conditions was performed using the signal of the secondary hadron $\mathrm{BCT}$. The uncertainty introduced by this WB background amounts to $\Delta \mathrm{R}^{\nu} / \mathrm{R}^{\nu}=0.3 \%$.

In normal running conditions the hadron beam is dumped at the end of the decay tunnel. In special runs with a dump at the entrance of that tunnel, the hadron beam is dumped there. The different location of the hadron dump has three consequences:

i) It introduces a different detection acceptance for the neutrinos created in the dump.

ii) The finite amount of gas in the $300 \mathrm{~m}$ long 'vacuum' acts as a target for secondary hadrons during normal data taking and neutrinos created in subsequent decays are not subtracted when the subtraction procedure is applied.

iii) The finite length of the special dump (1.5 m Fe or $\leq 8$ interaction lenghs) makes it necessary to apply a correction for non-containment and subsequent decay into neutrinos of secondary particles created in the dump and escaping from it.

Table 5 shows the background contamination expected in the detector for each condition of running. Errors are statistical only. The corrections were included in the WB background.

\subsubsection{Cosmic-ray background}

The uncertainty introduced by the cosmic-ray background during normal data taking is small, because sufficient data were taken in periods without beam to measure the correction and also because the correction is rather small. The cosmic-ray contribution to CC events is negligible and is $1 \%$ of the NC sample in the neutrino beam, and $4 \%$ of the NC sample in the antineutrino beam.

\subsection{Determination of the cross-section ratios}

The ratio of $\mathrm{NC}$ to $\mathrm{CC}$ events and the $\bar{\nu}$ to $\nu \mathrm{CC}$ cross-section ratio were determined for different values of the cut in hadron energy. This makes it possible to study the dependence of the value of $\sin ^{2} \theta_{\mathrm{w}}$ on the hadron energy cut. 
The corrections applied to obtain the sample of physical events in the neutrino beam are given in Table 6 for a $4 \mathrm{GeV}$ hadron energy cut. The effects of mixing of $\mathrm{NC}$ and $\mathrm{CC}$ events and the subtraction of $\nu_{\mathrm{e}}$-induced events from $\mathrm{K}_{\mathrm{e} 3}$ decays in the beam are also given. The results for the antineutrino data are given in Table 7.

A correction for a small deviation from isoscalarity of the target material was applied to the ratios of NC to CC events to derive the cross-section ratios, $R^{\nu}$ and $R^{\bar{\nu}}$. For the ratio $r$ of the total antineutrino and neutrino $\mathrm{CC}$ cross-sections, $\mathrm{r}$, an additional correction for the different spectra in neutrino and antineutrino exposures was applied. This is because, Eq. (9) being formulated in terms of differential cross-sections, any integrated cross-section satisfies the equation for arbitrary regions in $\mathrm{x}$ and $\mathrm{y}$, provided the integral is taken over the same neutrino and antineutrino spectrum.

The measurements of $R^{v}, R^{\bar{\nu}}$, and $\mathrm{r}$ are given for 4 and $9 \mathrm{GeV}$ hadron energy cuts in Table 8 .

\section{DETERMINATION OF $\sin ^{2} \theta$}

The electroweak mixing angle was deduced from Eq. (10). It is valid for an isoscalar target containing only $\mathrm{u}, \overline{\mathrm{u}}$, and $\mathrm{d}, \overline{\mathrm{d}}$ quarks without quark mixing and must therefore be corrected for contributions from $\mathrm{s}$ and $\mathrm{c}$ quarks in the nucleon and for $\mathrm{c}$ quark production by quark mixing. These corrections were determined in the context of the quark model of the nucleon. Quark mixing was described by the Kobayashi-Maskawa matrix.

We applied radiative corrections to the ratios of the cross-sections, $R^{\nu}, R^{\vec{\nu}}$, and $r$.

Quark fragmentation in the final state of deep inelastic neutrino scattering leads to thresholds in the invariant mass which depend on the quark content of the reaction (see Table 9). The muon mass is explicitly taken into account in the expression for the $\mathrm{CC}$ reaction.

\subsection{Radiative corrections}

We applied radiative corrections to $R^{\nu}, R^{\bar{\nu}}$, and r, following Bardin et al. [20], in the on-shell renormalization scheme of Sirlin [21]. In this scheme the calculable electroweak observables can be expressed in terms of the following set of independent quantities:

i) $\alpha$, the fine structure constant at $\mathrm{m}_{\mu}^{2}\left(\alpha^{-1}=137.035963\right)$;

ii) $\mathrm{G}_{\mathrm{F}}^{\mu}$, the Fermi constant in $\mu$ decay $\left(\mathrm{G}_{\mathrm{F}}^{\mu}=1.16637 \times 10^{-5} \mathrm{GeV}^{-2}\right)$;

iii) $\mathrm{m}_{\mathrm{Z}}$ and $\mathrm{m}_{\mathrm{H}}$, the physical masses of the $Z^{0}$ and Higgs bosons;

iv) $\left(\mathrm{m}_{\mathrm{i}}\right)$, the set of fermion masses.

$\sin ^{2} \theta_{\mathrm{w}}$ is defined from the measured $\mathrm{W}$ and $\mathrm{Z}$ masses (e.g. in $\mathrm{p} \overline{\mathrm{p}}$ colliders) by the expression:

$$
\sin ^{2} \theta_{\mathrm{w}}=1-\mathrm{m}_{\mathrm{w}}^{2} / \mathrm{m}_{\mathrm{Z}}^{2}
$$

The theoretical uncertainty on $\sin ^{2} \theta_{\mathrm{w}}$ due to the unknown value of the mass of the top quark $\left(30 \mathrm{GeV} / \mathrm{c}^{2}<\mathrm{m}_{\mathrm{t}}<240 \mathrm{GeV} / \mathrm{c}^{2}\right)$ and of the Higgs boson $\left(10 \mathrm{GeV} / \mathrm{c}^{2}<\mathrm{m}_{\mathrm{H}}<1000 \mathrm{GeV} / \mathrm{c}^{2}\right)$ is estimated to be \pm 0.0015 [22]. In the calculation the top and Higgs masses were set to $45 \mathrm{GeV}$ and $100 \mathrm{GeV}$, respectively.

One-loop electroweak radiative corrections were calculated using a set of computer programs made available to us by Bardin. The calculation was done in four steps.

The double differential cross-sections $\mathrm{d}^{2} \sigma / \mathrm{dxdy}$ for scattering of $\nu(\bar{\nu})$ off individual quarks are first calculated up to one-loop corrections. The initial quark momentum is then replaced by $\zeta \mathrm{p}_{\mathrm{N}}$, where $\zeta$ is the fraction of nucleon four-momentum $\mathrm{p}_{\mathrm{N}}$ carried by the parton; $\zeta$ is the Nachtmann definition of the scaling variable taking the finite target mass into account.

The cross-sections were then multiplied by the parton distribution functions $f(\zeta)$ of individual quarks given by Barger and Phillips [23], integrated over $\zeta$ and incoherently summed for all types of quarks involved in the reaction. 
Diagrams of internal bremsstrahlung can generate real photon emission. Owing to the detection method, the energy of these photons adds to the shower energy. We took the relative detector response to electromagnetic and hadronic showers in the ratio of 1.17 into account [17] in calculating this part of the radiative corrections. The effective energy threshold $(4 \mathrm{GeV})$ for $\mathrm{CC}$ events is shifted with respect to that for $\mathrm{NC}$ events.

Finally, a weighted average of cross-sections over protons and neutrons was calculated.

Tables of corrections to NC and CC double differential cross-sections were produced and integrated over the experimental conditions using a linear interpolation method. The correction to $\sin ^{2} \theta_{\mathrm{w}}$ is -0.0092 .

It must be pointed out that the calculation of radiative corrections to semi-leptonic neutrino interactions is model dependent owing to the somewhat arbitrary choice of structure functions used and the initial and final quark-mass dependence. Bardin et al. checked that the dependence on the structure functions was weak. They also studied the effect of a variation of the final quark masses between $1 \mathrm{MeV}$ and $1 \mathrm{GeV}$ and found that it changed the corrections on $\mathrm{CC}$ and NC cross-sections by less than $1 \%$.

The radiative corrections were also calculated using computer programs made available to us by Roos et al. [24]. This calculation was performed using different structure functions and gave a correction to $\sin ^{2} \theta_{\mathrm{w}}$ of -0.0122 . More calculations were done by other groups [11] taking into account similar experimental conditions. They give reasonable agreement with the previous ones. We therefore estimate an uncertainty on the correction of $\Delta \sin ^{2} \theta_{\mathrm{w}}= \pm 0.002$.

\subsection{Quark-model corrections}

The ratio of the contributions to the cross-sections given by scattering off antiquarks and quarks was modelled by requiring consistency with the measurement of $r$ in this analysis; we found

$$
\overline{\mathrm{q}} / \mathrm{q}_{\mathrm{v}}=0.19 \pm 0.02
$$

where $\overline{\mathrm{q}}$ and $\mathrm{q}_{\mathrm{v}}$ denote, as usual, the momentum weighted contents of antiquarks and valence quarks in the nucleon, respectively. We described the strange-quark sea using experimental data on neutrino-induced dimuon production [25] and used

$$
\bar{s} / \mathrm{d}_{\mathrm{v}}=0.06 \pm 0.02
$$

Following Ref. [26], we parametrized the $\mathrm{x}$ dependence of the strange sea by the expression

$$
\mathrm{s}\left(\mathrm{x}, \mathrm{Q}^{2}\right)=\mathrm{A}(1-\mathrm{x})^{\mathrm{B}}+\mathrm{C} \exp (-\mathrm{Dx})
$$

using $\mathrm{Q}^{2}$-dependent values of the parameters $\mathrm{A}, \mathrm{B}, \mathrm{C}$, and $\mathrm{D}$. For $\mathrm{Q}^{2}=5$ and $20 \mathrm{GeV}^{2}$ the parameters are given in Table 10. This parametrization is consistent with the dimuon analysis of Ref. [25].

For the charm-quark sea we used

$$
\mathrm{c} / \mathrm{s}=0.2 \pm 0.2
$$

as derived from dimuon production by deep inelastic muon scattering [27]. In flavour-changing reactions $(\mathrm{d} \rightarrow \mathrm{c}, \mathrm{s} \rightarrow \mathrm{c}$ ) we described the kinematical threshold effects introduced by the $\mathrm{c}$ quark mass $m_{c}$ using the so-called slow rescaling model [19] with a fixed value of $m_{c}=1.5 \mathrm{GeV} / \mathrm{c}^{2}$. This mass is not directly determined from measurements and has an uncertainty which is estimated to be 
$\pm 0.3 \mathrm{GeV} / \mathrm{c}^{2}$. This is the largest theoretical uncertainty affecting $\sin ^{2} \theta_{\mathrm{w}}$ derived from Eq. (10). We therefore give $\sin ^{2} \theta_{\mathrm{w}}$ for this central mass value and a linear approximation valid in the range $1 \mathrm{GeV} / \mathrm{c}^{2}<\mathrm{m}_{\mathrm{c}}<2 \mathrm{GeV} / \mathrm{c}^{2}$. We hope that further progress on determining $\mathrm{m}_{\mathrm{c}}$ can be made, thus enabling a more precise determination of $\sin ^{2} \theta_{\mathrm{w}}$.

We used values of the Kobayashi-Maskawa (K-M) mixing matrix derived assuming three families of quarks and requiring unitarity of the matrix [28].

Finally, we corrected Eq. (10) for effects due to the longitudinal structure function $F_{\mathrm{L}}$ and used a parametrization consistent with QCD predictions and with available measurements [13].

Higher twist effects are claimed to be small [12]; nevertheless, we investigated their effects by varying the hadron energy cut from $4 \mathrm{GeV}$ to $9 \mathrm{GeV}$ (see subsection 5.4).

\subsection{Discussion}

The different corrections applied to obtain $\sin ^{2} \theta_{\mathrm{w}}$ from $\mathrm{R}^{\nu}$ and $\mathrm{r}$ measured with a $4 \mathrm{GeV}$ energy cut are given in Table 11. Although the net correction is small, it is made of large single contributions.

An estimation of the uncertainties introduced by the model-dependent corrections ('theoretical uncertainties') is also given. The major part of the uncertainties is introduced by the uncertainty in the c-quark mass $\mathrm{m}_{\mathrm{c}}$. The uncertainty in the normalization of the s-quark component of the cross-section cancels to a large extent for values of $\mathrm{m}_{\mathrm{c}}$ near $1.5 \mathrm{GeV}$, because it contributes comparable fractions to the $\mathrm{NC}$ and $\mathrm{CC}$ cross-sections.

\subsection{Results}

Applying both quark-parton model (qpm) and radiative (rad) corrections, we obtain the final result

$$
\sin ^{2} \theta_{\mathrm{W}}=0.236+0.012\left(\mathrm{~m}_{\mathrm{c}}-1.5\right) \pm 0.005(\mathrm{exp} .) \pm 0.003 \text { (theor.) }
$$

where $m_{c}$ is the mass of the charm quark in $\mathrm{GeV} / \mathrm{c}^{2}$. The total theoretical uncertainty in the determination of $\sin ^{2} \theta_{\mathrm{w}}$, excluding the uncertainty on $\mathrm{m}_{\mathrm{c}}$, is estimated to be of the order of \pm 0.003 .

Higher twist effects were neglected. To investigate their effects we repeated the analysis for hadron energies larger than $9 \mathrm{GeV}$; the values of $\sin ^{2} \theta_{\mathrm{w}}$ are given in Table 12. In each case the 'raw' value is given, by simply substituting the measurements of $R^{\nu}$ and $r$ in Eq. (10), and in addition the values after radiative corrections only and after radiative and quark-model corrections. The difference between the two values of $\sin ^{2} \theta_{\mathrm{w}}$ is consistent with the statistical uncertainty of the event sample between 4 and $9 \mathrm{GeV}$ hadron energy, combined with the different systematic errors from the hadron-energy threshold. The value of $\sin ^{2} \theta_{\mathrm{w}}$ does not vary with the hadron-energy threshold, thus demonstrating the consistency of the methods used.

We can use the measurement of $R^{\bar{\nu}}$ to investigate the consistency with the assumption of $e=1$. Figure 15 shows the quark-model prediction for $R^{\nu}$ and $R^{\bar{\nu}}$ for a range of values of $\sin ^{2} \theta_{\mathrm{W}}$. The shaded area along the curve indicates the theoretical uncertainty. For $\sin ^{2} \theta_{\mathrm{W}}=0.236$, the value derived from $\mathrm{R}^{\nu}$ and $\mathrm{r}$, a range of $\varrho$ values is indicated as well. We note good agreement with the model for $\varrho=1.030 \pm 0.020$.

This result can be compared with those obtained in other semileptonic neutrino-scattering experiments. Starting from a previous measurement (1981) of $\mathrm{R}^{\nu}$ and $\mathrm{r}$ by this Collaboration [7], and applying the same quark-parton model and radiative corrections, we obtained $\sin ^{2} \theta_{\mathrm{w}}=0.223 \pm$ 0.014 (exp.) (see Table 1). Our new result is compared with those obtained in other recent semileptonic neutrino-scattering experiments (see Table 13) for $\mathrm{m}_{\mathrm{c}}=1.5 \mathrm{GeV} / \mathrm{c}^{2}$. The FMMF Collabaration at FNAL used a method similar to ours, classifying NC and CC interactions on an 
event-by-event basis [29]. The CDHS Collaboration at CERN [30] and the CCFR Collaboration at FNAL [31] used a statistical method for classification based on the total event length. The agreement is good and significant considering the differences in experimental procedures used. The mean value derived from these experiment is

$$
\sin ^{2} \theta_{\mathrm{W}}^{\text {average }}=0.233 \pm 0.004 \text { (exp.) } \pm 0.005 \text { (theor.) }
$$

\section{DISCUSSION AND CONCLUSIONS}

Using the new precise value of $\sin ^{2} \theta_{\mathrm{w}}$ together with the recent direct measurements of $\mathrm{m}_{\mathrm{W}}$ and $m_{z}[9,10]$ we have determined the electroweak radiative shift of the $W$ mass from Eq. (6) and find

$$
\Delta \mathrm{r}=0.077 \pm 0.025 \text { (exp.) } \pm 0.038 \text { (syst.) }
$$

in good agreement with the calculated correction of $\Delta \mathrm{r}=0.0713 \pm 0.0013$ assuming $\mathrm{m}_{\mathrm{t}}=45 \mathrm{GeV}$ and $m_{H}=100 \mathrm{GeV}[1,11]$. The systematic error of $\Delta \mathrm{r}$ is the quadratic sum of the theoretical uncertainties in determining $\sin ^{2} \theta_{\mathrm{W}}$ from semileptonic neutrino scattering and of the energy scale error entering into the $\mathrm{W}$ and $\mathrm{Z}^{0}$ mass determinations. The agreement between theory and experiment is very good. The value of $\sin ^{2} \theta_{\mathrm{w}}$ obtained from deep inelastic $\nu_{\mu}$ scattering without electroweak radiative corrections differs from the uncorrected value obtained from $\mathrm{m}_{\mathrm{W}}$ and $\mathrm{m}_{\mathrm{Z}}$ (see Table 1) by $\Delta \sin ^{2} \theta_{\mathrm{W}}=0.027 \pm 0.011$, where experimental, systematic and theoretical errors are combined quadratically. This difference is significant at the $2.5 \mathrm{\sigma}$ level and illustrates quite nicely the existence of and need for radiative corrections. Assuming, therefore, their validity a precise determination of the $\varrho$ parameter can be obtained using the relation

$$
\varrho^{2}=\mathrm{R}^{\nu} / \mathrm{R}^{\mathrm{calc}}
$$

where $R^{\text {calc }}$ is calculated with Eq. (10) using the weighted average of $\sin ^{2} \theta_{\mathrm{W}}$ measurements by the UA1 [9] and UA2 [10] Collaborations working at CERN of $\sin ^{2} \theta_{\mathrm{W}}=0.226 \pm 0.008$ and $\mathrm{R}^{\nu}$ is corrected for radiative and quark-parton model corrections. Propagating the errors quadratically we find

$$
\varrho=0.990-0.013\left(\mathrm{~m}_{\mathrm{c}}-1.5\right) \pm 0.009(\mathrm{exp} .) \pm 0.003 \text { (theor.) }
$$

in good agreement with the minimal Standard Model $\left(\mathrm{m}_{\mathrm{c}}\right.$ is the mass of the charm quark in $\left.\mathrm{GeV} / \mathrm{c}^{2}\right)$.

New physics appendages to the Standard Model or a different input value for $m_{t}$ would modify the prediction of Eqs. (4) and (5). We can therefore use the good agreement with experiment as a constraint. For example, if $\mathrm{m}_{\mathrm{t}}$ were $250 \mathrm{GeV} / \mathrm{c}^{2} \Delta \mathrm{r}$ would become zero, whereas $\sin ^{2} \theta_{\mathrm{W}}$ hardly changes [32]. From the present experimental value of $\Delta r$, Marciano and Sirlin [33] find the constraint.

$$
\mathrm{m}_{\mathrm{t}} \leq 180 \mathrm{GeV}
$$

In the case of a heavy fourth-generation charged lepton $\mathrm{L}$ with massless neutrino companion the corresponding bound is [32]

$$
\mathrm{m}_{\mathrm{L}} \leq 300 \mathrm{GeV}
$$


Future high-precision experiments should determine $m_{W}$ and $m_{Z}$ to $\pm 0.1 \mathrm{GeV}$ and $\pm 0.02 \mathrm{GeV}$, respectively and, combined with the result of a determination of $\sin ^{2} \theta_{\mathrm{W}}$ from $\nu_{\mu} \mathrm{e}$ and $\bar{\nu}_{\mu} \mathrm{e}$ scattering in the CHARM II experiment to \pm 0.005 without theoretical uncertainty, should thus provide even better constraints or perhaps a hint of new physics.

Our final comment is concerned with the comparison of $\sin ^{2} \theta_{\mathrm{w}}$ predicted by Grand Unified Theories. The experimental result presented here contradicts the prediction of the minimal SU(5) model [1,34],

$$
\sin ^{2} \theta_{\mathrm{w}}=0.214 \pm 0.004 \quad[\mathrm{SU}(5)]
$$

which has already been ruled out by the experimental lower limit of proton decay [35]. Equation (16) can be used to constrain the additional mass scales of non-minimal Grand Unified Theories. For example, in the case of supersymmetric theories (SUSY), the experimental result is in good agreement with the mass constraint $\mathrm{M}_{\text {SusY }} \geq 1 \mathrm{TeV}$ [32].

\section{Acknowledgements}

We should like to thank our numerous technical collaborators most sincerely for their enthusiastic and competent assistance which ensured the excellent performance of the neutrino detector with its associated systems. The accumulation of the large data sample in this experiment has been made possible owing to the dedicated operation of the CERN Super Proton Synchrotron and its Neutrino Facility. The detector has been built and operated with financial support from NIKHEF (Amsterdam), The Bundesministerium für Technologie and Forschung (Bonn), the Instituto Nazionale di Fisica Nucleare (Rome), and from the Institute of Theoretical and Experimental Physics (Moscow), which we wish to acknowledge. We are indebted to D.Yu Bardin, C.H. Llewellyn Smith, O. Nachtmann, R. Petronzio, M. Roos and A. Sirlin for discussions on the theoretical interpretation of our results and to members of the CDHS Collaboration for discussions about the quark-parton model. 


\section{REFERENCES}

[1] For a recent review see, for example, U. Amaldi et al., preprint CERN-EP/87-93, to be published in Phys. Rev. D.

[2] M.A. Bouchiat and C.A. Piketty, J.Phys. (France) 46, 1897 (1985).

S.Gilbert, N. Noecker, R. Watts and C. Wiemann, Phys. Rev. Lett. 55, 2680 (1985).

[3] C.Y. Prescott et al., Phys. Lett 77B, 347 (1978) and 84B, 524 (1979).

[4] F. Bergsma at al., CHARM Collaboration, Phys. Lett 147B, 481 (1984).

[5] L.A. Ahrens et al., Phys. Rev. Lett. 54, 18 (1985).

[6] K. Abe et al., Phys. Rev. Lett. 56, 1107 (1986).

[7] M. Jonker at al., CHARM Collaboration, Phys. Lett. 99B, 265 (1981).

[8] H. Abramowicz et al., CDHS Collaboration, Z. Phys. C28, 51 (1985).

[9] S. Geer, UA1 Collaboration, Proc. Int. Conf. on High Energy Physics, Berkeley, 1986 (World Scientific, Singapore, 1987), vol. 2, p. 982.

[10] R. Ansari et al., UA2 Collaboration, Phys. Lett. 190B, 238 (1987).

[11] W.J. Marciano and A. Sirlin, Phys. Rev. D22, 2695 (1980), and Erratum Phys. Rev. D31, 213 (1985); Nucl. Phys. B189, 442 (1981).

J.F. Weather and C.H. Llewellyn Smith, Nucl. Phys. 208, 27 (1982) and Erratum Nucl. Phys. B226, 547 (1983).

[12] C.H. Llewellyn Smith, Nucl. Phys. B228, 205 (1983)

[13] See, for example, a recent review by M. Diemoz, F. Ferroni and E. Longo, Phys. Rep. 130, 293 (1986).

[14] A. Grant and J.M. Maugain, Internal Report CERN/EF/BEAM 83-2.

[15] J. Panman, Contribution to Workshop on SPS Fixed-Target Physics in the years 1984-1989, CERN 83-02 (1980), p. 80.

[16] A.N. Diddens et al., CHARM Collaboration, Nucl. Instrum. Methods 178, 27 (1980).

C. Bosio et al.,Nucl. Instrum. Methods 157, 35 (1978) .

M. Jonker et al., CHARM collaboration, Nucl. Instrum. Methods 200, 183 (1982).

[17] J. Dorenbosch et al., CHARM Collaboration, Nucl. Instrum. Methods, A253, 203 (1987).

[18] M. Jonker et al., CHARM Collaboration, Phys. Lett. 107B, 241 (1981).

[19] R.M. Barnett, Phys. Rev. D14, 70 (1976);

H. Georgi and H. D. Politzer, Phys. Rev. D14, 1829 (1976).

[20] D.Yu. Bardin and O.M. Dokuchaeva, preprint JINR-E2-86-260 (1986);

D.Yu. Bardin and O.M. Federenko, Yad. Phys. 30, 811 (1979) [Transl. Sov. J. Nucl. Phys. 30, 418 (1979)].

D.Yu. Bardin and O.M. Dokuchaeva, Yad. Phys. 36, 482 (1982) [Transl.: Sov. J. Nucl. Phys. 36, 282 (1982)].

[21] A. Sirlin, Phys. Rev. D22, 971 (1980).

[22] R.G. Stuart, Z. Phys. C34, 445 (1987).

[23] V. Barger and R.J.N. Phillips, Nucl. Phys. B73, 269 (1974).

[24] I. Liede, E.A. Paschos, M. Roos and S. Sakakibara, Helsinki University preprint HU-TFT-83-45.

I. Liede, M. Roos and S. Sakakibara, Helsinki University preprint, HU-TFT-84-28.

[25] J. Knobloch et al., CDHS Collaboration, Private communication.

[26] H. Abramowicz et al., CDHS Collab., Z. Phys. C17, 283 (1983).

[27] J.J. Aubert et al., EMC Collaboration, Nucl. Phys. B213, 31 (1983).

[28] K. Kleinknecht and B. Renk, Phys. Lett. 130B, 459 (1983) .

[29] D. Bogert et al., FMMF Collaboration, Phys. Rev. Lett. 55, 1969 (1985). 
[30] H. Abramowicz et al., CDHS Collaboration, Phys. Rev. Lett. 57, 298 (1986).

[31] P. Reutens et al., CCFRR Collaboration, Phys. Lett. 152B, 404 (1985).

[32] W. J. Marciano and A. Sirlin (to be published).

W. J. Marciano, Proc. 23rd Int. Conf. on High Energy Physics, Berkeley, 1986 (World Scientific, Singapore, 1987), vol. 2, p. 999.

[33] W.J. Marciano and A. Sirlin, Phys. Rev. D29 945, (1984) and Erratum Phys. Rev. D31, 213 (1985).

[34] W. J. Marciano, A. Sirlin, Phys. Rev. Lett. 46, 163 (1981).

[35] M. Goldhaber, Proc 23rd Int. Conf. on High Energy Physics, 1986, Berkeley (World Scientific, Singapore, 1987), vol. 1, p. 248. 
Table 1

Values of $\sin ^{2} \theta_{\mathrm{w}}$ obtained from various processes and the values after electroweak radiative corrections have been applied

\begin{tabular}{|l|l|l|l|}
\hline \multicolumn{1}{|c|}{ Experiment } & $\sin ^{2} \theta_{\mathrm{W}}^{\text {unc }}$ & \multicolumn{1}{c|}{ Condition } & $\sin ^{2} \theta_{\mathrm{W}}^{\text {corr }}$ \\
\hline Parity violation in Cs atoms [2] & $0.221 \pm 0.027$ & & $0.228 \pm 0.027$ \\
eD scattering asymmetry [3] & $0.224 \pm 0.020$ & $\varrho=1$ & $0.218 \pm 0.020$ \\
$\nu_{\mu}\left(\bar{\nu}_{\mu}\right)$ e scattering [4] & $0.215 \pm 0.034$ & independent of $\varrho$ & $0.215 \pm 0.034$ \\
$\nu_{\mu}\left(\bar{\nu}_{\mu}\right)$ e scattering [5] & $0.209 \pm 0.032$ & independent of $\varrho$ & $0.209 \pm 0.032$ \\
$\nu_{\mu}\left(\bar{\nu}_{\mu}\right)$ p scattering [6] & $0.220 \pm 0.016$ & $\mathrm{M}_{\mathrm{A}}=1.05 \mathrm{GeV}$ & $0.220 \pm 0.016$ \\
$\nu_{\mu} \mathrm{N}$ deep inel. scattering [7] & $0.232 \pm 0.014$ & $\varrho=1$ & $0.223 \pm 0.014$ \\
$\nu_{\mu} \mathrm{N}$ deep inel. scattering [8] & $0.239 \pm 0.012$ & $\varrho=1$ & $0.226 \pm 0.012$ \\
$\mathrm{~m}_{\mathrm{W}}$ and $\mathrm{m}_{\mathrm{Z}}[9]$ & $0.219 \pm 0.015$ & $\varrho=1$ & $0.214 \pm 0.016$ \\
$\mathrm{~m}_{\mathrm{W}}$ and $\mathrm{m}_{\mathrm{Z}}[10]$ & $0.215 \pm 0.008$ & $\varrho=1$ & $0.232 \pm 0.008$ \\
\hline
\end{tabular}

Table 2

Exposures and dead-times

\begin{tabular}{|l|r|r|r|r|r|}
\cline { 2 - 5 } \multicolumn{1}{c|}{} & $\nu$ & $\nu$-dump & $\bar{\nu}$ & $\bar{\nu}$-dump & GATE 2 \\
\hline p.o.t. for data collection $\left(10^{16}\right)$ & 338.0 & 113.2 & 97.8 & 49.0 & - \\
p.o.t. selected $\left(10^{16}\right)$ & 326.9 & 92.3 & 90.2 & 47.0 & - \\
Dead-times $(\%):$ & & & & & \\
- unweighted & 10.0 & 6.7 & 8.4 & 8.0 & 6.0 \\
- flux weighted & 16.5 & 10.7 & 14.0 & 14.9 & - \\
- double events & 3.0 & 1.1 & 1.2 & 0.4 & 0.1 \\
Average dead-time (\%) & 19.0 & 11.8 & 15.0 & 15.3 & 6.1 \\
& & & & & \\
p.o.t. corrected for dead-time $\left(10^{16}\right)$ & 264.7 & 81.4 & 76.7 & 39.8 & - \\
& & & & & \\
Integrated gate-time corrected & & & & & \\
$\quad$ for dead-time (s) & 177.7 & 53.1 & 53.1 & 24.1 & 806.0 \\
\hline
\end{tabular}


Table 3

Systematic errors on the $\nu_{\mu}$ and $\bar{\nu}_{\mu}$ flux ratio (\%)

\begin{tabular}{|l|c|}
\hline \multicolumn{1}{|c|}{ Source } & $\begin{array}{c}\text { Error on flux ratio } \\
(\%)\end{array}$ \\
\hline Monte Carlo statistics & 1.0 \\
K/ $\pi$ ratio & 0.9 \\
Beam width & 0.2 \\
Beam steering & 0.1 \\
Vertex resolution & 0.1 \\
Muon tracking & 0.4 \\
SSD position & 0.5 \\
& \\
Trapped muons & 0.7 \\
SSD stability & 1.0 \\
Dead-time & 0.5 \\
\hline Total error (sum of squares) & 2.0 \\
\hline
\end{tabular}

Table 4

Classification matrix of a sample of $5 \%$ of the data. Horizontal rows give the program classification; vertical columns give the scanning classification. Events are classified as $\mathrm{CC}, \mathrm{NC}$, and events outside the fiducial volume ( $\mathrm{OCC}, \mathrm{ONC})$.

\begin{tabular}{|l|r|r|r|r|}
\hline & CC & \multicolumn{1}{|c|}{$\mathrm{NC}$} & OCC & ONC \\
\hline $\mathrm{CC}$ & 5669 & 1 & 0 & 0 \\
$\mathrm{NC}$ & 1 & 2171 & 0 & 0 \\
OCC & 3 & 0 & 2728 & 0 \\
ONC & 0 & 2 & 0 & 1593 \\
\hline
\end{tabular}




\section{Table 5}

Contamination from dump and other sources

\begin{tabular}{|c|c|c|c|c|}
\hline & $\nu$ & $\nu$-dump & $\bar{\nu}$ & $\bar{\nu}$-dump \\
\hline $\begin{array}{l}\text { From dump }\left(\times 10^{-3}\right) \\
\text { From rest gas }\left(\times 10^{-5}\right) \\
\text { From shielding at end of } \\
\text { decay tunnel }\left(\times 10^{-5}\right)\end{array}$ & $\begin{array}{c}- \\
2.2 \pm 1.0 \\
11.0 \pm 1.2\end{array}$ & $\begin{array}{c}4.1 \pm 2.1 \\
-\end{array}$ & $\begin{array}{c}- \\
2.0 \pm 1.0 \\
9.0 \pm 1.0\end{array}$ & $\begin{array}{c}1.3 \pm 0.7 \\
-\end{array}$ \\
\hline Total $\left(\times 10^{-3}\right)$ & $0.13 \pm 0.02$ & $4.1 \pm 2.1$ & $0.11 \pm 0.2$ & $1.3 \pm 0.7$ \\
\hline
\end{tabular}

Table 6

Event numbers for neutrino exposure $\left(E_{h} \geq 4 \mathrm{GeV}\right)$

\begin{tabular}{|l|c|c|}
\cline { 2 - 3 } \multicolumn{1}{c|}{} & NC & CC \\
\hline Uncorrected data sample & $39239 \pm 198$ & $108472 \pm 329$ \\
Trigger + filter efficiency & $7 \pm 4$ & $0 \pm 0$ \\
Scan correction & $40 \pm 40$ & $60 \pm 44$ \\
Corrected raw data sample & $39286 \pm 202$ & $108532 \pm 332$ \\
& & \\
WB and cosmic correction & $-2310 \pm 87$ & $-4311 \pm 119$ \\
- of which WB & $-1998 \pm 88$ & $-4308 \pm 119$ \\
- of which cosmic & $-312 \pm 8$ & $-3 \pm 1$ \\
Clean NBB data sample & $36976 \pm 225$ & $104220 \pm 361$ \\
& & \\
Possible difference in energy & - & $0 \pm 129$ \\
cut for NC and CC & $-3737 \pm 105$ & $3735 \pm 105$ \\
Lost muons & $1893 \pm 50$ & $-1835 \pm 50$ \\
$\pi$ and K decay & $-1768 \pm 68$ & $-106 \pm 6$ \\
$\mathrm{~K}_{\mathrm{e} 3} \mathrm{CC}$ & $-532 \pm 20$ & $-33 \pm 2$ \\
$\mathrm{~K}_{\mathrm{e} 3}$ NC & & \\
& & \\
Corrected event numbers & $32831 \pm 283$ & $105981 \pm 408$ \\
\hline
\end{tabular}


Table 7

Event numbers for antineutrino exposure $\left(E_{h} \geq 4 \mathrm{GeV}\right)$

\begin{tabular}{|l|r|r|}
\cline { 2 - 3 } \multicolumn{1}{c|}{} & NC & CC \\
\hline Uncorrected data sample & $2314 \pm 48$ & $5455 \pm 74$ \\
Trigger + filter efficiency & $1 \pm 1$ & $0 \pm 0$ \\
Scan correction & $0 \pm 0$ & $0 \pm 0$ \\
Corrected raw data sample & $2315 \pm 48$ & $5455 \pm 74$ \\
WB and cosmic correction & $-368 \pm 26$ & $-450 \pm 29$ \\
- of which WB & $-275 \pm 26$ & $-449 \pm 29$ \\
- of which cosmic & $-93 \pm 3$ & $-1 \pm 0$ \\
Clean NBB data sample & $1947 \pm 55$ & $5005 \pm 79$ \\
& & \\
Difference in energy cut & - & $0 \pm 16$ \\
Lost muons & $-45 \pm 5$ & $45 \pm 5$ \\
$\pi$ and K decay & $104 \pm 5$ & $-98 \pm 6$ \\
$K_{\mathrm{e} 3}$ CC & $-56 \pm 3$ & $-3 \pm 1$ \\
$\mathrm{~K}_{\mathrm{e} 3}$ NC & $-19 \pm 1$ & $-1 \pm 0$ \\
Corrected event numbers & $1931 \pm 58$ & $4948 \pm 81$ \\
\hline
\end{tabular}

\section{Table 8}

Ratios $\mathbf{R}^{\nu}, \mathbf{R}^{\bar{\nu}}$ and $\mathrm{r}$ for two $\mathrm{E}_{\mathrm{h}}$ thresholds

\begin{tabular}{|c|c|c|c|}
\hline $\mathrm{E}_{\mathrm{h}}$ cut & $\mathrm{R}^{\nu}$ & $\mathrm{R}^{\bar{\nu}}$ & $\mathrm{r}$ \\
\hline $4 \mathrm{GeV}$ & $0.3093 \pm 0.0031$ & $0.390 \pm 0.014$ & $0.456 \pm 0.011$ \\
$9 \mathrm{GeV}$ & $0.3052 \pm 0.0033$ & $0.397 \pm 0.015$ & $0.429 \pm 0.010$ \\
\hline
\end{tabular}


Table 9

Mass thresholds

\begin{tabular}{|l|c|c|}
\hline Final-state quark & $\mathrm{CC}$ & $\mathrm{NC}$ \\
\hline u or d quark & $\mathrm{m}_{\mathrm{N}}+\mathrm{m}_{\pi}$ & $\mathrm{m}_{\mathrm{N}}+\mathrm{m}_{\pi}$ \\
s quark & $\mathrm{m}_{\mathrm{N}}+\mathrm{m}_{\mathrm{K}}$ & $\mathrm{m}_{\mathrm{N}}+2 \mathrm{~m}_{\mathrm{K}}$ \\
c quark & $\mathrm{m}_{\mathrm{N}}+\mathrm{m}_{\mathrm{D}}$ & $\mathrm{m}_{\mathrm{N}}+2 \mathrm{~m}_{\mathrm{D}}$ \\
\hline
\end{tabular}

Table 10

Strange sea parameters

\begin{tabular}{|c|c|c|}
\hline Parameter & $\mathrm{Q}^{2}=5 \mathrm{GeV}^{2}$ & $\mathrm{Q}^{2}=20 \mathrm{GeV}^{2}$ \\
\hline $\mathrm{A}$ & 0.0551 & 0.0933 \\
$\mathrm{~B}$ & 6.3907 & 7.1820 \\
$\mathrm{C}$ & 0.0671 & 0.0637 \\
$\mathrm{D}$ & 13.21 & 16.12 \\
\hline
\end{tabular}

Table 11

Corrections to $\sin ^{2} \theta_{\mathrm{w}}\left(\mathrm{E}_{\mathrm{h}} \geq 4 \mathrm{GeV}\right)$

\begin{tabular}{|l|c|c|}
\hline \multicolumn{1}{|c|}{ Source } & $\Delta \sin ^{2} \theta_{\mathrm{w}}$ & $\begin{array}{l}\text { Theoretical } \\
\text { uncertainty }\end{array}$ \\
\hline Muon mass & +0.0011 & \pm 0.0001 \\
$\mathrm{~W}^{2}$ thresholds, $\mathrm{F}_{\mathrm{L}}$ & +0.0005 & \pm 0.0005 \\
$\mathrm{~K}-\mathrm{M}$ mixing matrix & & \pm 0.0010 \\
Strange sea for $\mathrm{m}_{\mathrm{c}}=0$ & -0.0074 & \pm 0.0010 \\
Charm sea for $\mathrm{m}_{\mathrm{c}}=0$ & +0.0015 & \pm 0.0010 \\
Radiative corrections & -0.0092 & \pm 0.0020 \\
Total uncertainty $\left(\mathrm{fixed} \mathrm{m}_{\mathrm{c}}\right)$ & +0.0140 & \pm 0.0030 \\
Charm mass $\left(\mathrm{m}_{\mathrm{c}}=1.5 \mathrm{GeV} / \mathrm{c}^{2}\right)$ & +0.0005 & \pm 0.0030 \\
\hline Total $\left(\mathrm{m}_{\mathrm{c}}=1.5 \mathrm{GeV} / \mathrm{c}^{2}\right)$ & & \\
\hline
\end{tabular}


Table 12

$\operatorname{Sin}^{2} \theta_{w}$ values as a function of the $E_{h}$ threshold

\begin{tabular}{|l|l|l|l|}
\hline$E_{\mathrm{h}}$ threshold & $\sin ^{2} \theta_{\mathrm{W}}^{\text {raw }}$ & $\sin ^{2} \theta_{\mathrm{W}}^{\mathrm{rad}}$ & $\sin ^{2} \theta_{\mathrm{W}}^{\mathrm{rad}}+\mathrm{qpm}$ \\
\hline $4 \mathrm{GeV}$ & 0.2356 & 0.2252 & $0.2359 \pm 0.0050$ \\
$9 \mathrm{GeV}$ & 0.2409 & 0.2302 & $0.2383 \pm 0.0052$ \\
\hline
\end{tabular}

Table 13

Comparison of values of $\sin ^{2} \theta_{\mathrm{w}}$ derived from semileptonic neutrino-scattering experiments.

The common theoretical error is \pm 0.003 .

\begin{tabular}{|l|l|c|}
\hline Group & Method & $\sin ^{2} \theta \mathrm{w}(\exp )$. \\
\hline FMMF [29] & Event-by-event & $0.246 \pm 0.016$ \\
CCFR [31] & Event length & $0.239 \pm 0.010$ \\
CDHS [30] & Event length & $0.225 \pm 0.005$ \\
CHARM & Event-by-event & $0.236 \pm 0.005$ \\
\hline
\end{tabular}


Figure captions

Fig. 1 Energy spectrum of neutrino flux.

Fig. 2 Schematic of the narrow-band-beam line.

Fig. 3 Hadron shower vertex resolution.

Fig. 4 Rejection of 'double events'.

Fig. 5 Trigger and filter efficiencies.

Fig. 6 Efficiency near the veto plane.

Fig. 7 a) Typical neutral-current and b) typical charged-current events recorded in the CHARM detector.

Fig. 8 Effective hadron shower length.

Fig. 9 Comparisons between Monte Carlo simulation and data of shower-energy distribution (a) and event radius (b) of $\mathrm{CC}$ events.

Fig. 10 Comparison between Monte Carlo simulation and data of average hadron shower length versus hadron shower energy (a) and distribution of measured muon total range (b) of CC events.

Fig. 11 Muon-finding efficiency.

Fig. 12 Dimuon production by neutrinos. Comparison of the model with data.

Fig. $13 \pi$ and $\mathrm{K}$ decay correction.

Fig. 14 Typical Cherenkov pressure curve in the neutrino beam.

Fig. 15 Quark-model prediction for $\mathbf{R}^{\nu}$ and $\mathbf{R}^{\bar{\nu}}$ as a function of $\sin ^{2} \theta_{\mathrm{W}}$ and $\varrho$. 


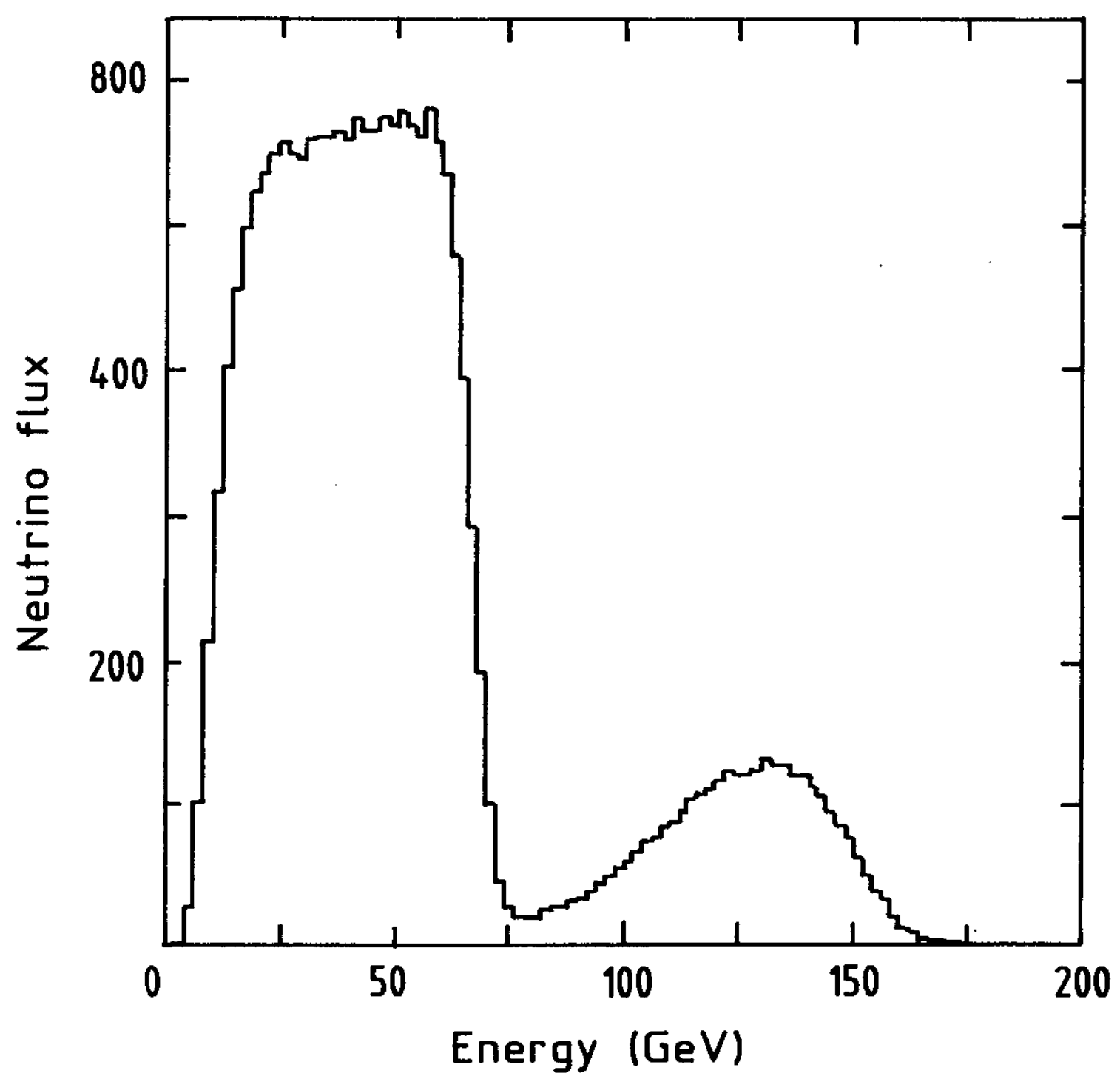

Fig. 1 


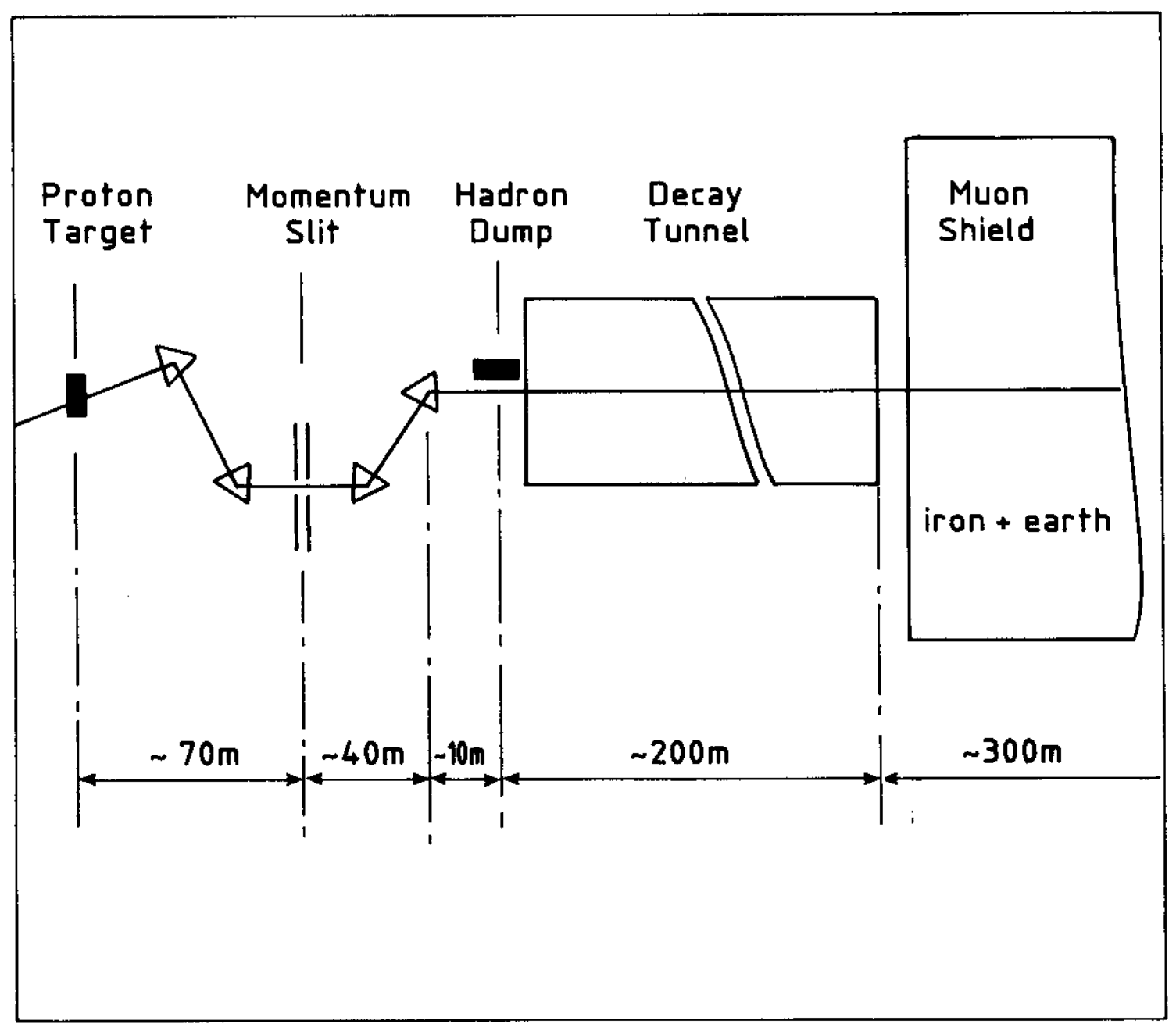

Fig. 2

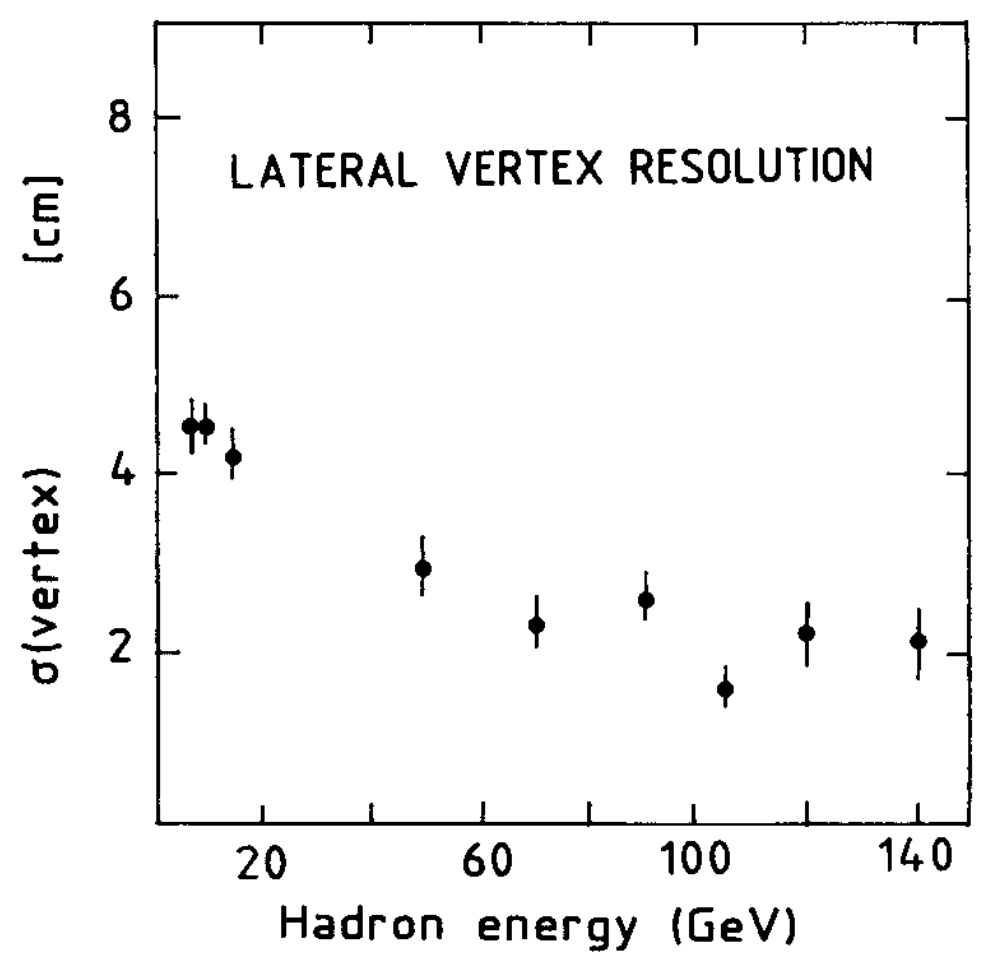

Fig. 3 


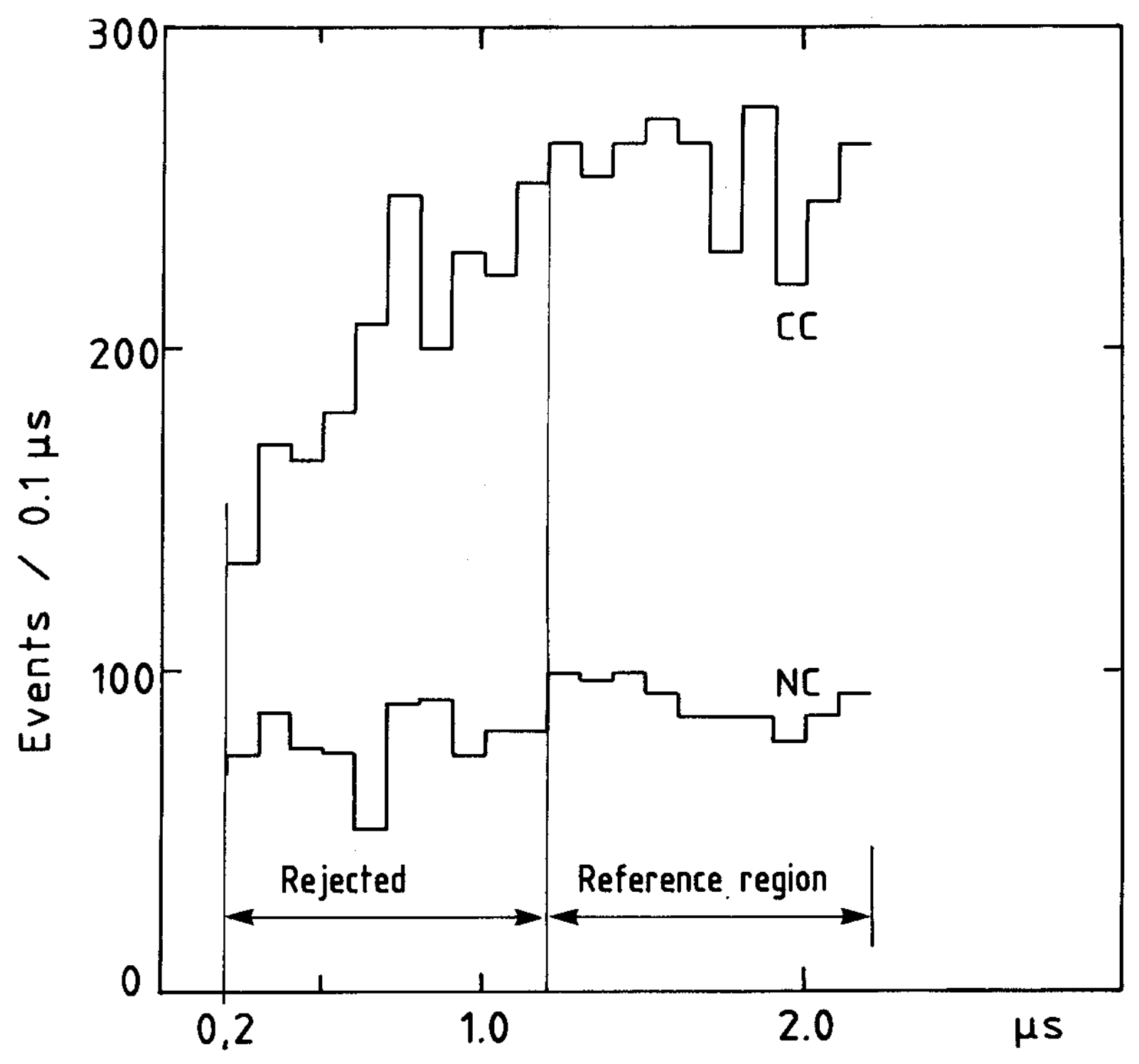

Time interval between successive events

Fig. 4 


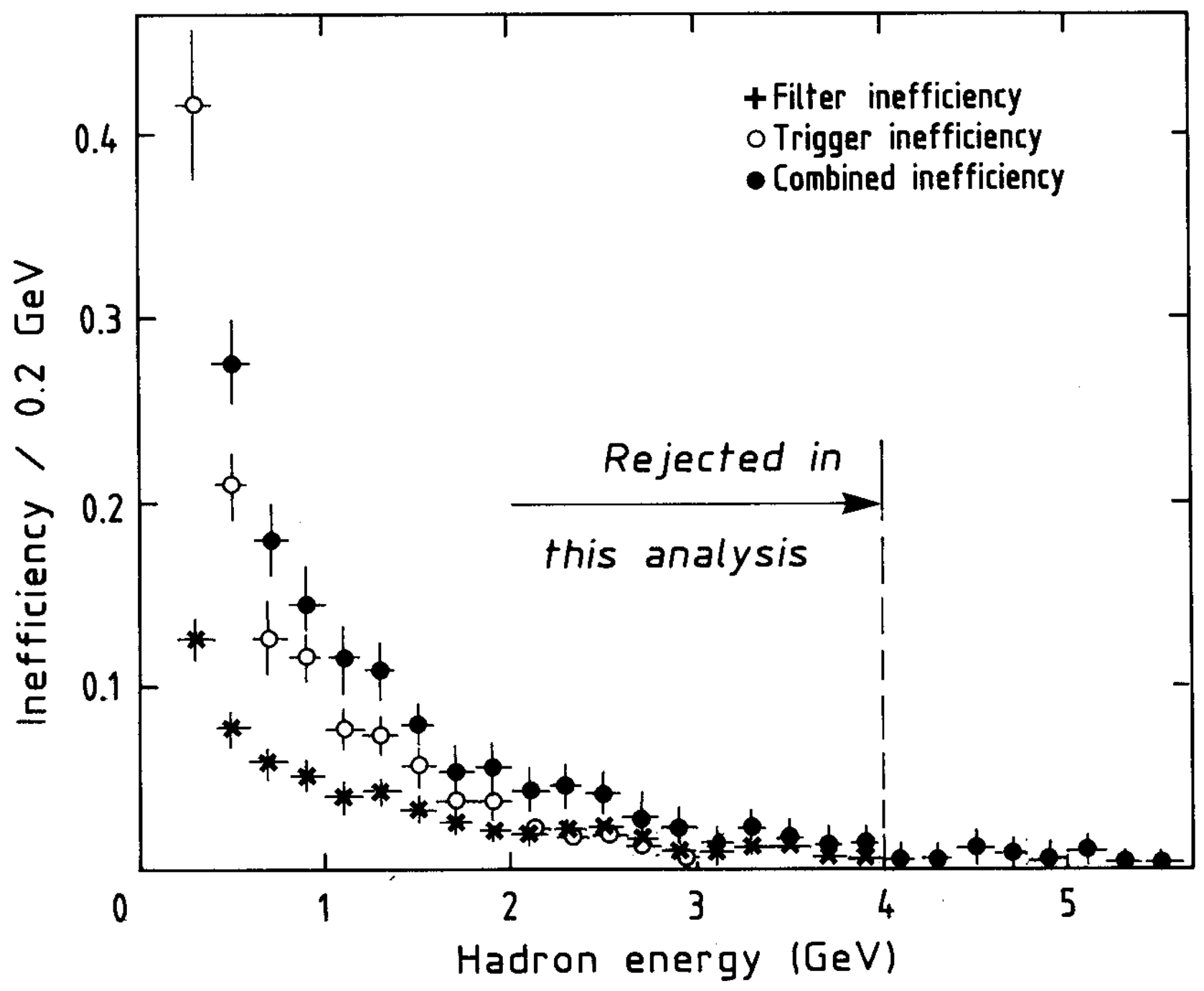

Fig. 5 


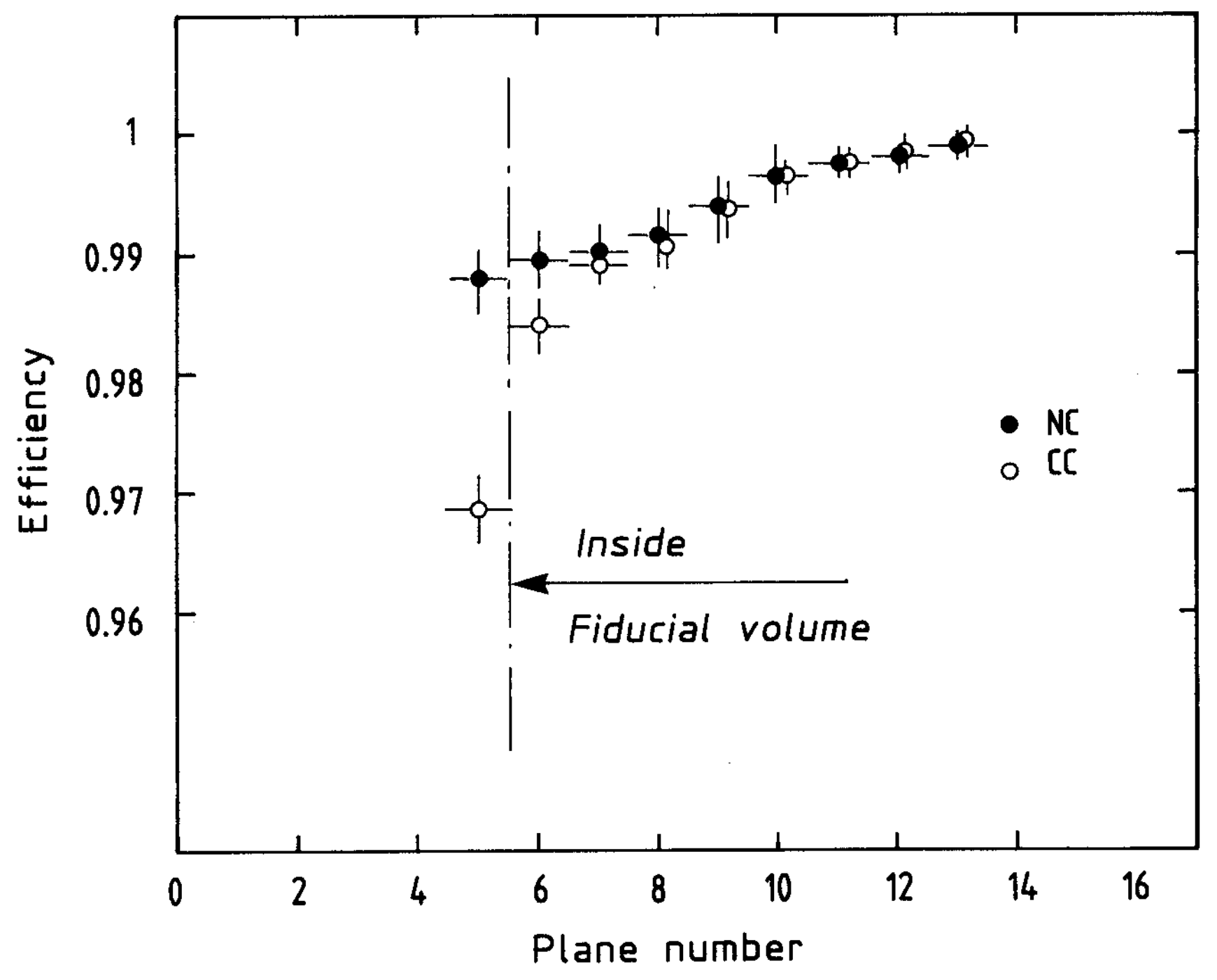

Fig. 6 

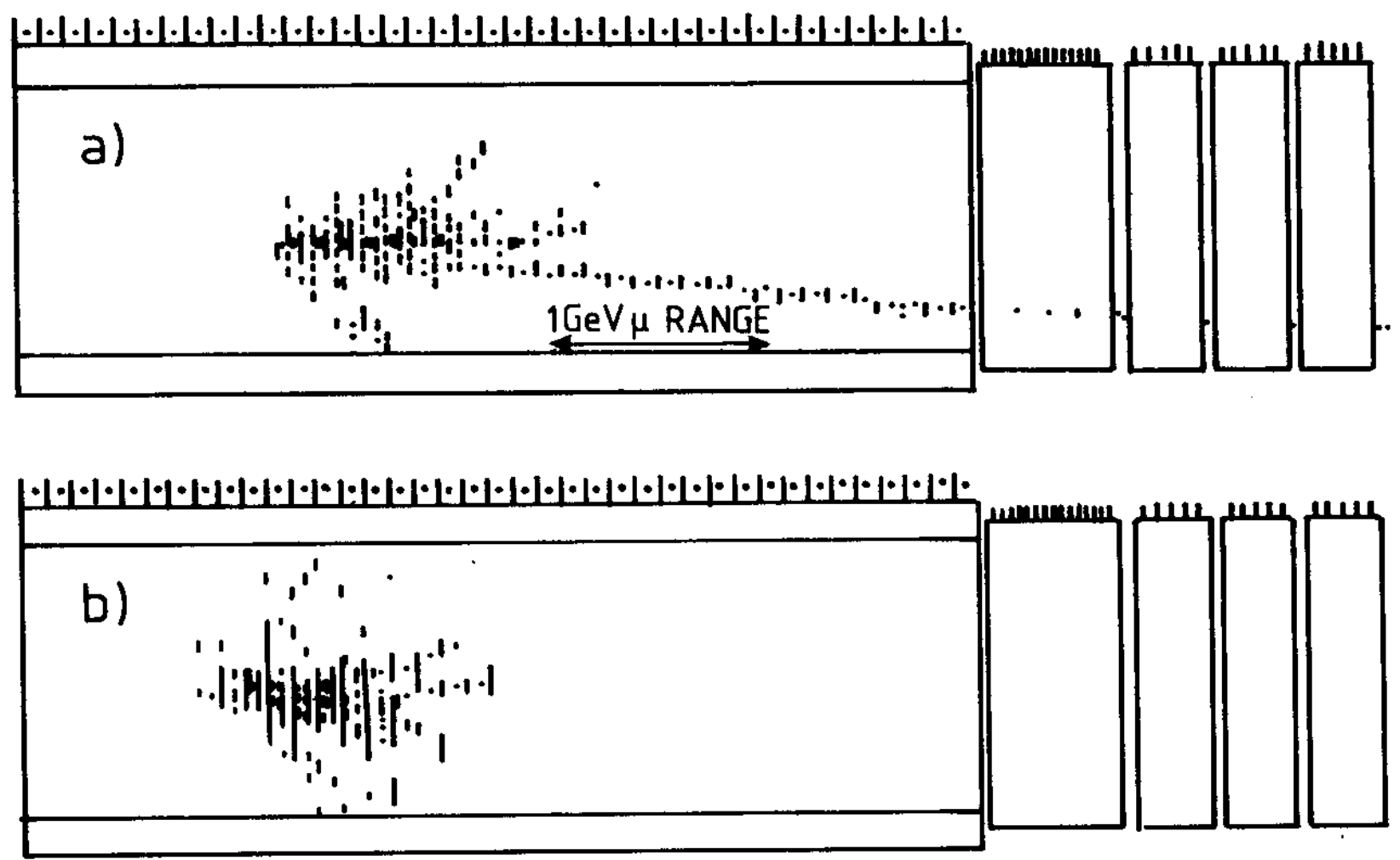

Fig. 7

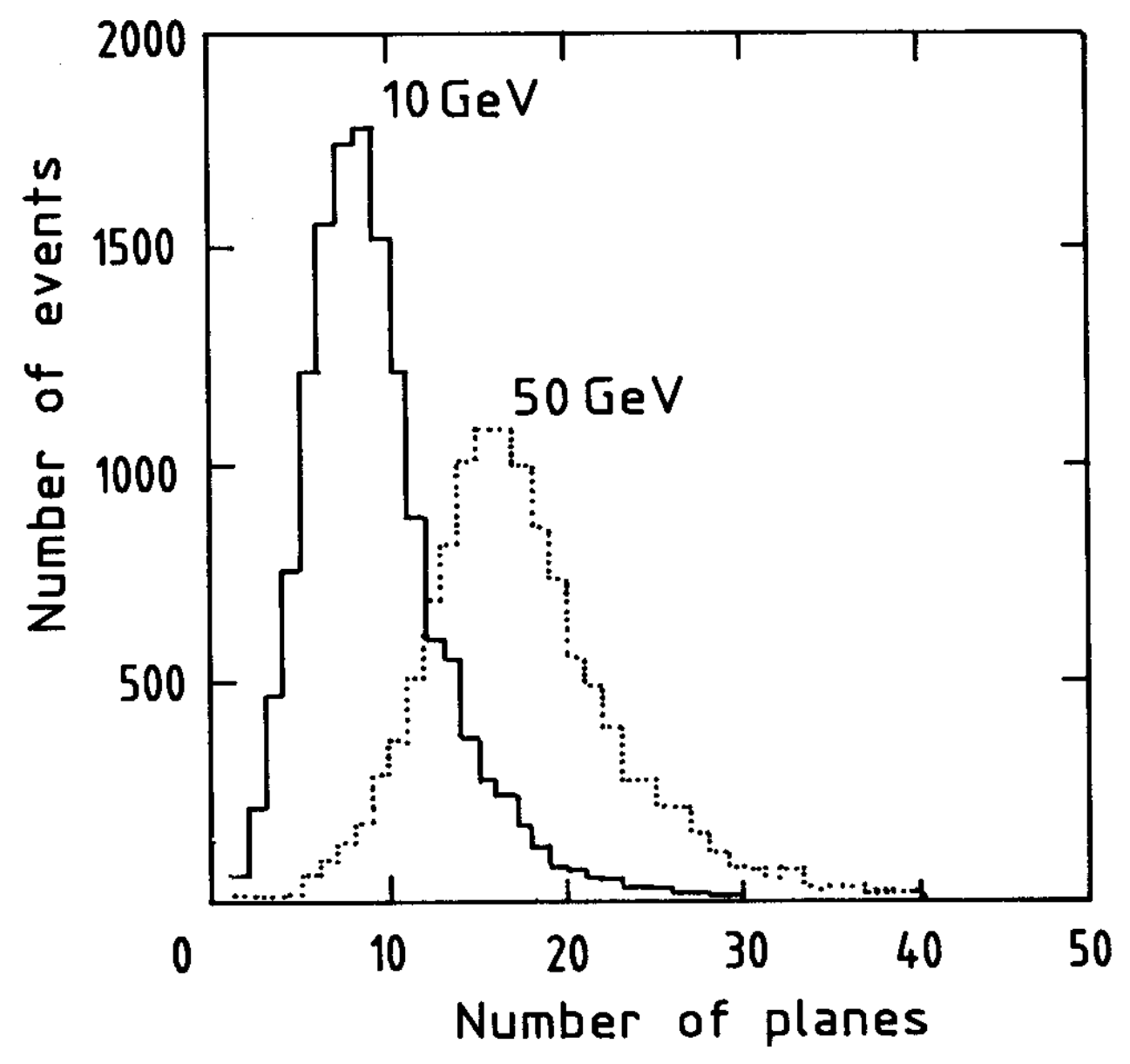

Fig. 8 

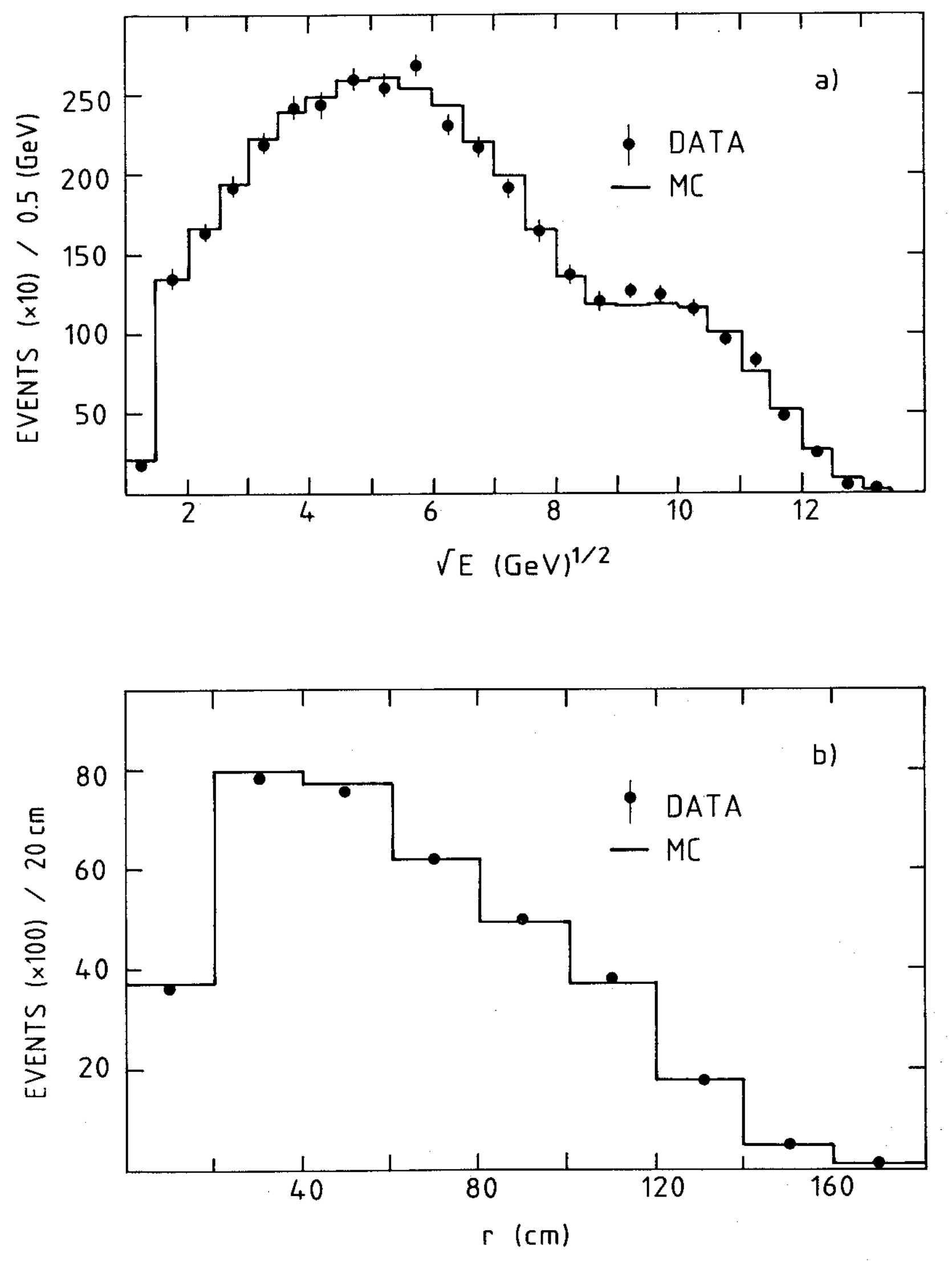

Fig. 9 

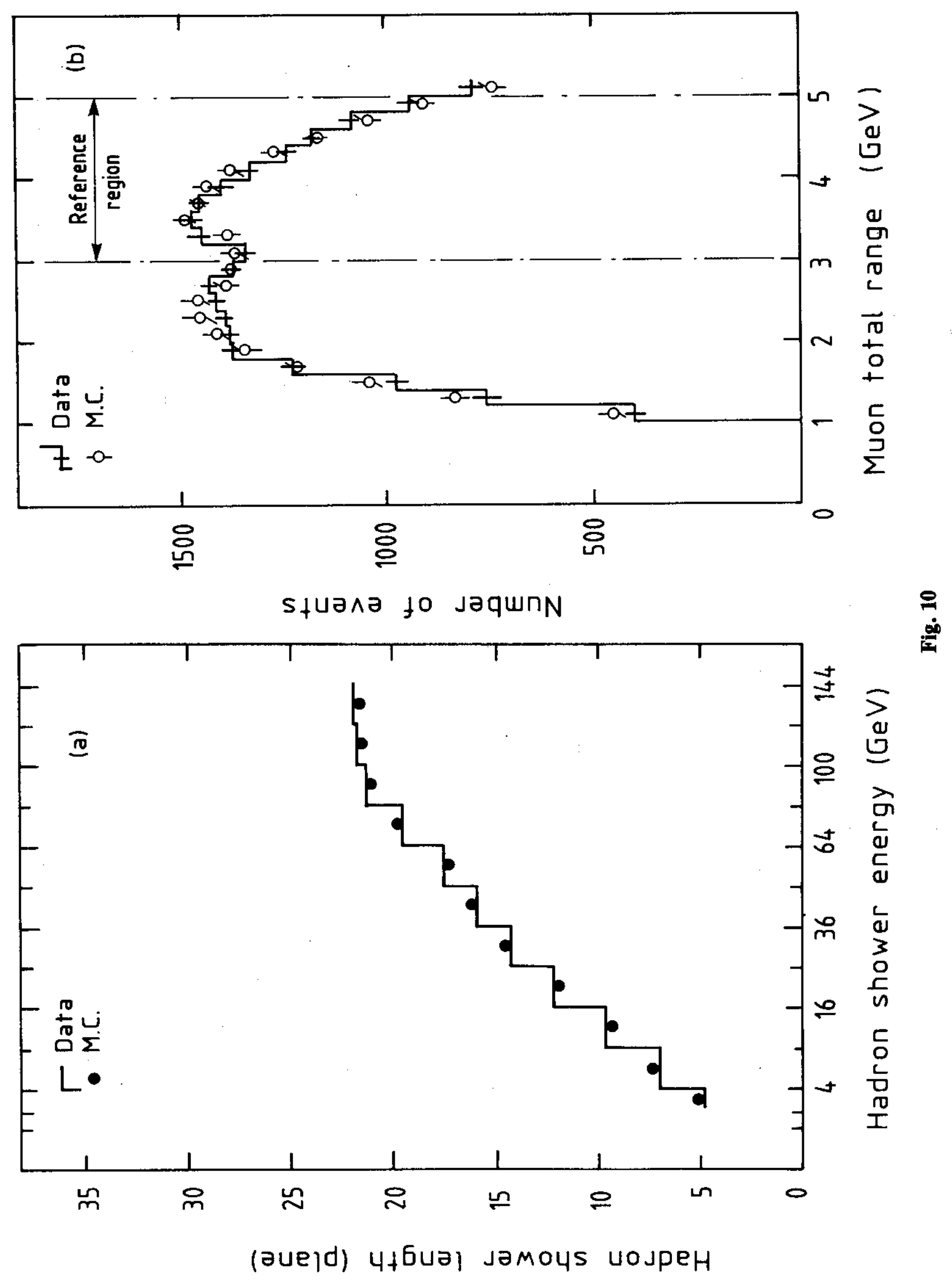


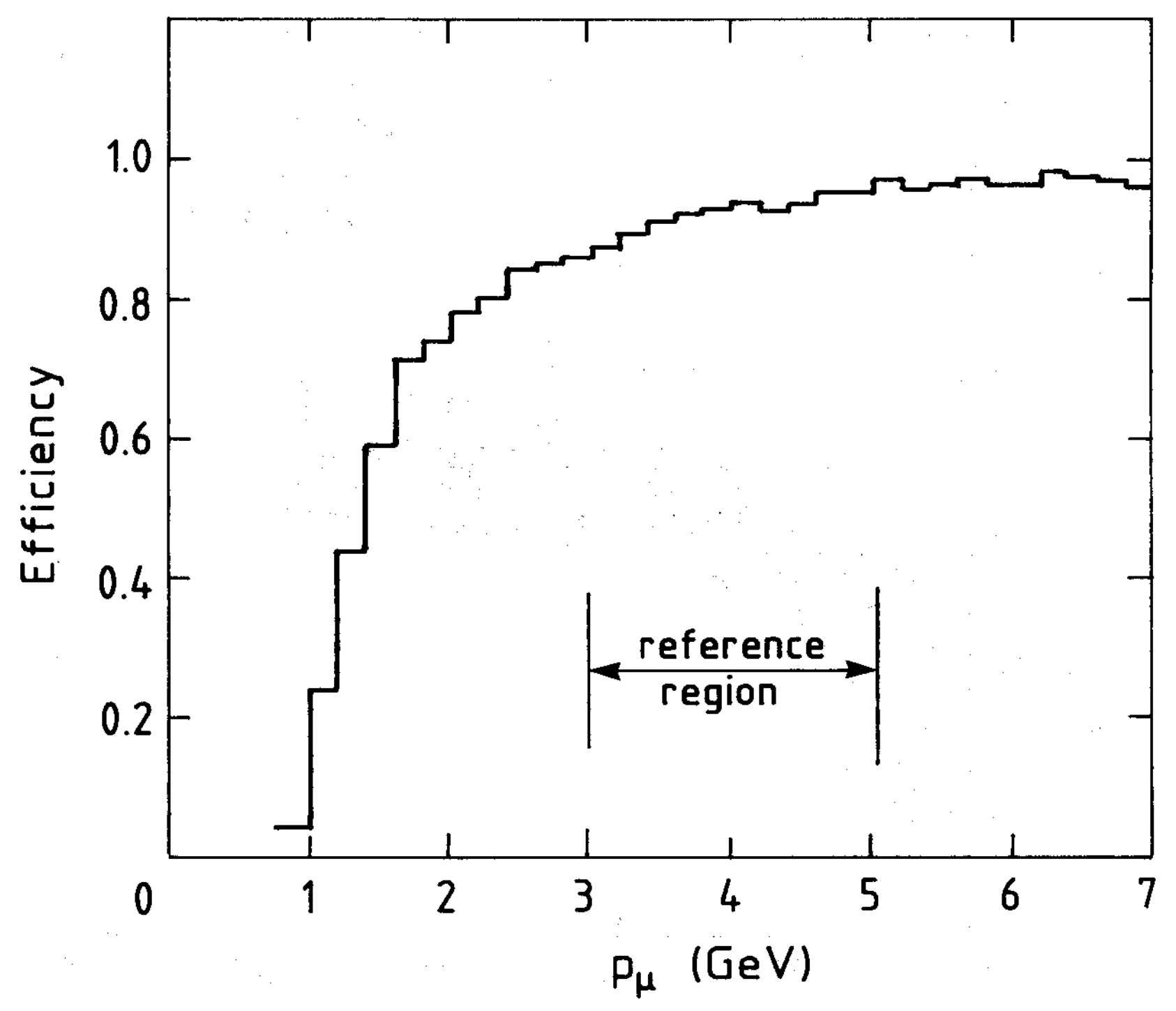

Fig. 11 


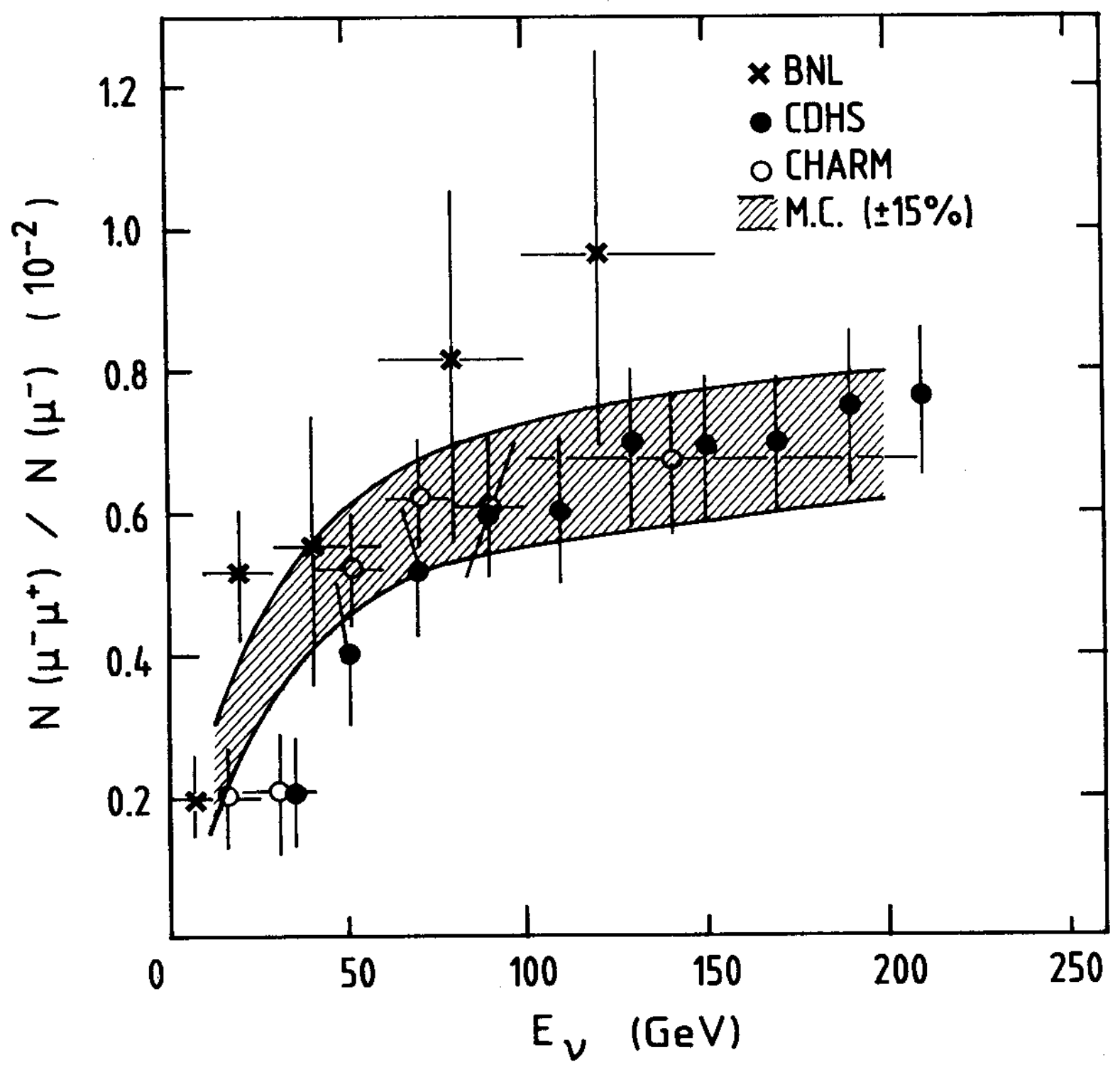

Fig. 12 


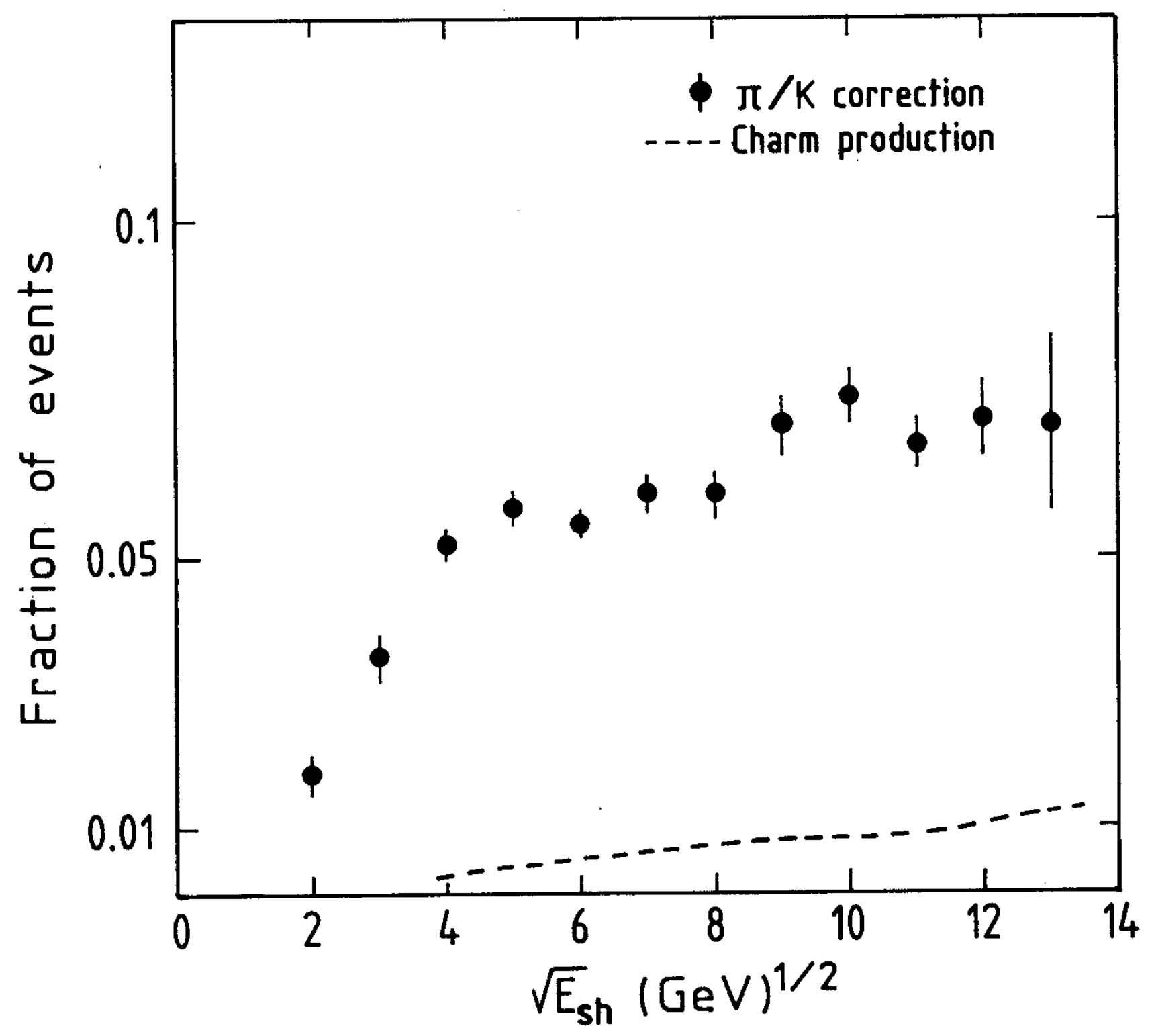

Fig. 13 


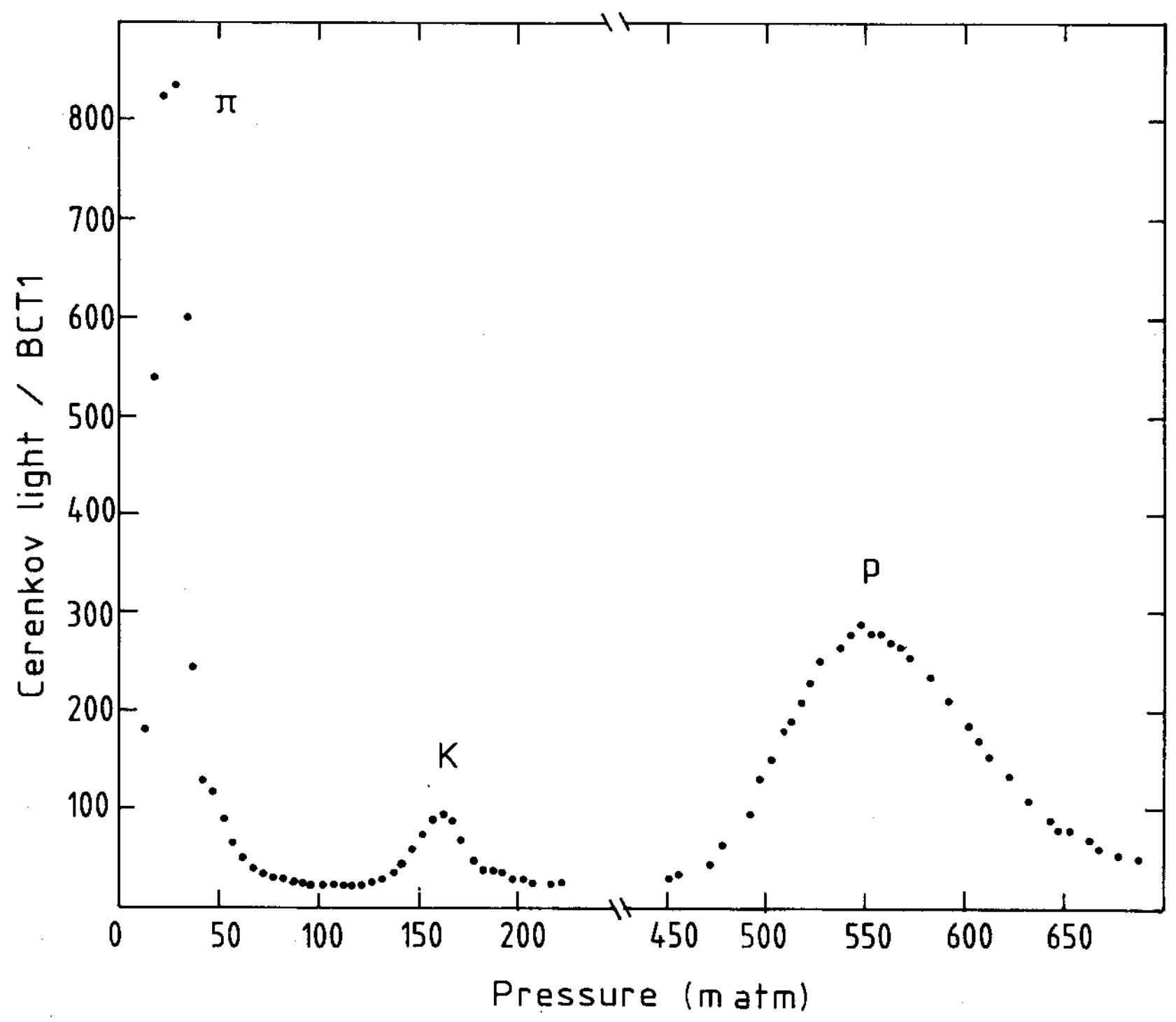

Fig. 14 


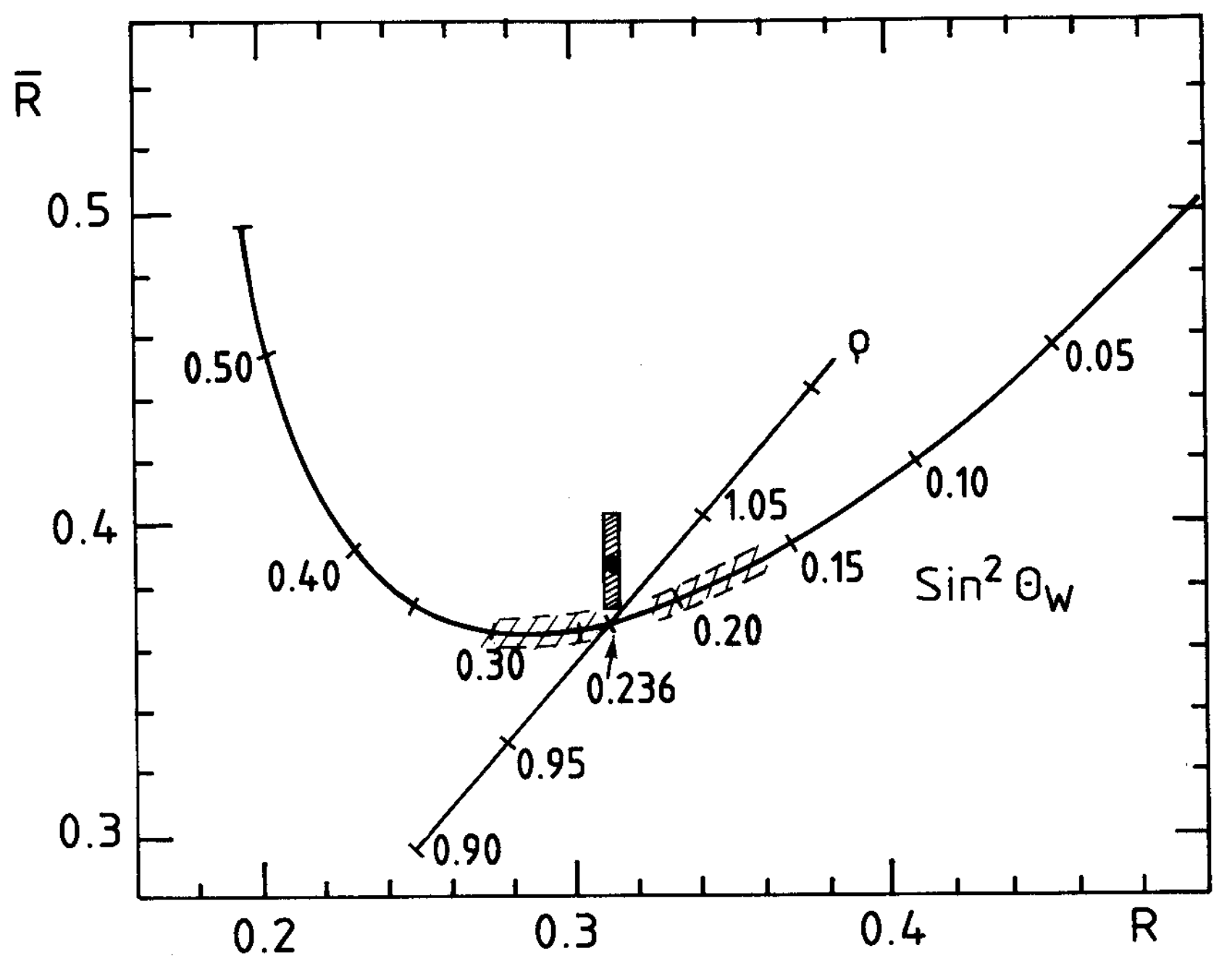

Fig. 15 